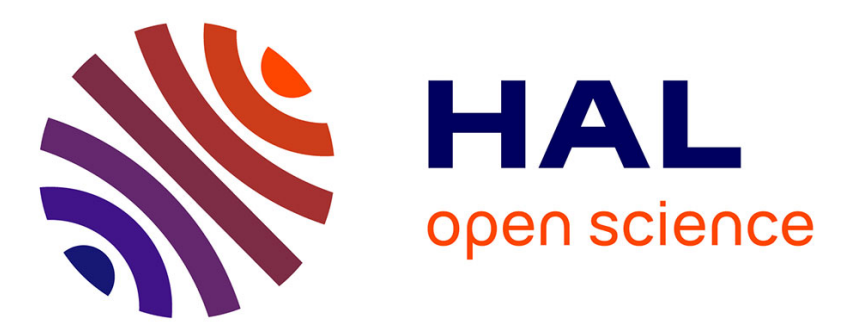

\title{
Dynamic Parameter Identification of Over-actuated Parallel Robots
}

\author{
Sébastien Briot, Sébastien Krut, Maxime Gautier
}

\section{To cite this version:}

Sébastien Briot, Sébastien Krut, Maxime Gautier. Dynamic Parameter Identification of Over-actuated Parallel Robots. Journal of Dynamic Systems, Measurement, and Control, 2015, 137 (11), pp.111002. 10.1115/1.4030867 . hal-01157978

\section{HAL Id: hal-01157978 https://hal.science/hal-01157978}

Submitted on 24 Jun 2019

HAL is a multi-disciplinary open access archive for the deposit and dissemination of scientific research documents, whether they are published or not. The documents may come from teaching and research institutions in France or abroad, or from public or private research centers.
L'archive ouverte pluridisciplinaire HAL, est destinée au dépôt et à la diffusion de documents scientifiques de niveau recherche, publiés ou non, émanant des établissements d'enseignement et de recherche français ou étrangers, des laboratoires publics ou privés. 


\title{
Dynamic Parameter Identification of Over-actuated Parallel Robots
}

\author{
Sébastien Briot ${ }^{1, *}$ \\ Maxime Gautier ${ }^{1,3}$ \\ ${ }^{1}$ Institut de Recherches en Communications et Cybernétique de Nantes (IRCCyN) \\ UMR CNRS 6597 - Nantes, France \\ \{Sebastien.Briot, Maxime.Gautier\}@irccyn.ec-nantes.fr \\ ${ }^{2}$ Laboratoire d'Informatique, de Robotique et de Microélectronique de Montpellier (LIRMM) \\ UMR CNRS 5506 - Montpellier, France \\ sebastien.krut@lirmm.fr \\ ${ }^{3}$ Nantes University, Nantes, France \\ ${ }^{*}$ Corresponding author \\ Tel: +33 (0)2 403769 58, Fax: +33 (0)2 40376930
}

May 29, 2015

\begin{abstract}
Off-line robot dynamic identification methods are based on the use of the Inverse Dynamic Identification Model (IDIM), which calculates the joint forces/torques (estimated as the product of the known control signal (the input reference of the motor current loop) with the joint drive gains) that are linear in relation to the dynamic parameters, and on the use of the linear least squares technique to calculate the parameters (IDIM-LS technique). However, as actuation redundant parallel robots are over-actuated, their IDIM has an infinity of solutions for the force/torque prediction, depending on the value of the desired overconstraint that is a priori unknown in the identification process. As a result, the IDIM cannot be used as it is for such a class of parallel robots.

This paper proposes a procedure for the dynamic parameter identification of actuation redundant parallel robots. The procedure takes advantage of two possible modified formulations for the IDIM of actuation redundant robots that can be used for identification purpose. The modified IDIM formulations project some or all input torques/forces onto the robot bodies, thus leading to a unique solution of the model that can then be used in the identification process. A systematic and straightforward way to compute these modified IDIM is presented. The identification of the inertial parameters of a planar parallel robot with actuation redundancy, the DualV, is then carried out using these modified IDIM. Experimental results show the validity of the methods.
\end{abstract}

\section{Introduction}

Parallel robots have increasingly been used for a few decades. This is due to their main advantages over serial counterparts that are: (i) higher intrinsic rigidity, (ii) larger payload-to-weight ratio, and (iii) higher velocity and acceleration capacities [1]. However, their main drawback is probably the presence of singularities in the workspace. In order to overcome this difficulty, actuation redundancy can be used $[2,3]$. Actuation redundancy occurs when normally passive joints are replaced by active ones. The robot becomes over-constrained. Overconstraints can be smartly used to improve the robot properties, such as increasing the acceleration or payload capacities [4] or even decreasing the backlash [5]. However, this involves the use of more complicated controllers. 


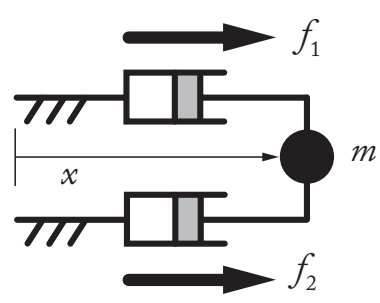

Figure 1: A one dof robot with actuation redundancy.

Several control approaches could be envisaged [6, 7], but it appears that, for high-speed robots or when varying loads have to be compensated (e.g. in pick-and-place operations or machining), computed torque control gives the best results $[5,8]$. This approach requires an accurate estimation of the dynamic model of the robot with the load [9]. However, a priori inertial parameters extracted from CAD are generally not given by the manufacturer, and even if they are given, (i) they may be inaccurate and (ii) friction terms inside the drive trains and passive joints are still unknown. Therefore, the identification of dynamic parameters is necessary.

Several schemes have been proposed in the literature to identify the dynamic parameters of robots $[10,11,12,13,14,15,16]$. Most of the dynamic off-line identification methods

- use an Inverse Dynamic Identification Model (IDIM) that gives linear relations between each joint force/torque and the dynamic parameters,

- build an over-determined linear system of equations obtained by sampling the IDIM while the robot is tracking some trajectories in position closed-loop control,

- estimate the parameter values using least squares techniques $(L S)$.

Good experimental results can be obtained if two main conditions are satisfied:

- joint velocities and accelerations are calculated by a well-tuned derivative band-pass filtering of joint position, and

- accurate values for joint drive gains are known to calculate the joint force/torque as the products of input references of the motor current loop and joint drive gains $[17,18,19]$.

For identifying the dynamic parameters of actuation redundant parallel robots, a major problem arises. To better understand it, let us consider the simple example shown in Fig. 1. This mechanism with 1 degree of freedom (dof) is moved through the use of two actuators mounted in parallel that can apply two independent forces denoted as $f_{1}$ and $f_{2}$ on the moving body of mass $m$. In this example, only the moving mass $m$ is considered (the robot legs are massless). For moving the mechanism, an infinity of possible forces exists, e.g. $\left[f_{1} f_{2}\right]=[m \ddot{x} 0]$, or also $\left[f_{1} f_{2}\right]=[0 m \ddot{x}]$, and even $\left[f_{1} f_{2}\right]=0.5[m \ddot{x} m \ddot{x}]$ or many other force combinations $\left[f_{1} f_{2}\right]=m[(1-\alpha) \ddot{x} \alpha \ddot{x}]$ for any value of $\alpha$. This is due to the fact that, for an over-actuated mechanism, the input efforts are not independent (indeed for this mechanism, we have $f_{1}=m \ddot{x}-f_{2}$ ) which leads to the fact that the generalized efforts are not the mechanism input efforts, but a linear combination of them.

It should be mentioned here that the function $c=\left|f_{1}-f_{2}\right|$ is called the overconstraint.

As a result, the mechanism IDIM would take the form $\left[f_{1} f_{2}\right]=\mathbf{I D M}_{\alpha} m$, where $\mathbf{I D M}_{\alpha}=$ $[(1-\alpha) \ddot{x} \alpha \ddot{x}]$ is the observation matrix. IDM $_{\alpha}$ depends on the parameter $\alpha$ which is a priori unknown. This issue prevents the use of the usual IDIM in the identification process as a unique formulation cannot be defined ${ }^{1}$. This problem also appears for any kind of actuation redundant parallel robots: their inverse dynamic model is not unique and depends of the overconstraint in the mechanism [5].

\footnotetext{
${ }^{1}$ It should be mentionned that in such a simple case, the value of the parameter $\alpha$ could be identified at each time interval, but this cannot be the case for a more general over-actuated parallel robot.
} 
It should be mentioned that the overconstraint could be fixed using a proper controller [20]. However, as shown by our own experience in the field, most of the time, manufacturers of industrial robots do not want to provide information on their controller, or even, in worst cases, the controller does not fix the overcontraint. In such cases, the value of the overconstraint cannot be considered as a priori known in the identification process as this quantity cannot be measured. Thus, identification using the usual IDIM for redundant parallel robots cannot be carried out.

To avoid the potential lack of information from the controller, another method should be proposed. Considering again the simple system presented in Fig. 1, it can be demonstrated that its dynamics can be uniquely described if the equations are projected not on the actuators as usual, but on the moving body such that: $f_{1}+f_{2}=m \ddot{x}$. Thus, by projecting the dynamic equations on a body different from the actuators, a modified IDIM formulation can be obtained. Contrary to the usual IDIM, this modified formulation does not express anymore the linear relations between each joint force and the dynamic parameters, but between a linear combination of the joint forces and the dynamic parameters. It should be mentioned that this formulation can be used in any case, the value of the overconstraint $c=\left|f_{1}-f_{2}\right|$ being known or not.

The aim of this paper is to generalize the approach to any kind of actuation redundant parallel robots and to propose a systematic and straightforward procedure for their dynamic parameter identification. This procedure is based on the computation of a modified IDIM, linear with respect to the inertial parameters, that does not require the knowledge of the overconstraint. Two different ways for computing the IDIM are proposed. These formulations also minimize the number of intermediate variables and operators used for the symbolic computation of the model. This point is crucial in identification as it minimizes the risk of error propagation (due to the measurement noise) in the computation of the observation matrix.

This systematic procedure is applied for the identification of a 3 dof planar parallel robot with actuation redundancy designed at the LIRMM and named the DualV [21].

A first condensed version of this paper has been proposed in [22]. Differences with the present paper are:

- a modified formulation is given for the computation of the IDIM of general parallel robots, in order to better explain the physical meaning of the equations.

- another new way to obtain an IDIM is shown and parameters estimated using this IDIM are compared with those obtained using the method developed in [22].

- new experiments and cross-validations have been performed and are presented in this paper.

\section{Inverse Dynamic Identification Model of Parallel Robots with no Actuation Redundancy}

This section presents some recalls on the computation of the IDIM of parallel robots without actuation redundancy. These recalls are necessary as the computation of the IDIM of actuation redundant parallel robots is partially based on some mathematical derivations shown in this part and to understand why the usual IDIM cannot be used in the identification procedure.

\subsection{Computation of the IDIM for Parallel Robots with no Actuation Redundancy}

A parallel robot is a complex multi-body system having several closed loops (Fig. 2(a)). It is composed of a moving platform connected to a fixed base by $n$ legs, each composed of $m_{i}$ elements. It is considered here that there is one actuator per leg, but the method can be easily extended to robots with several actuators for each leg.

The computation of IDIM of parallel robots with no actuation redundancy is decomposed into two steps [23]: 


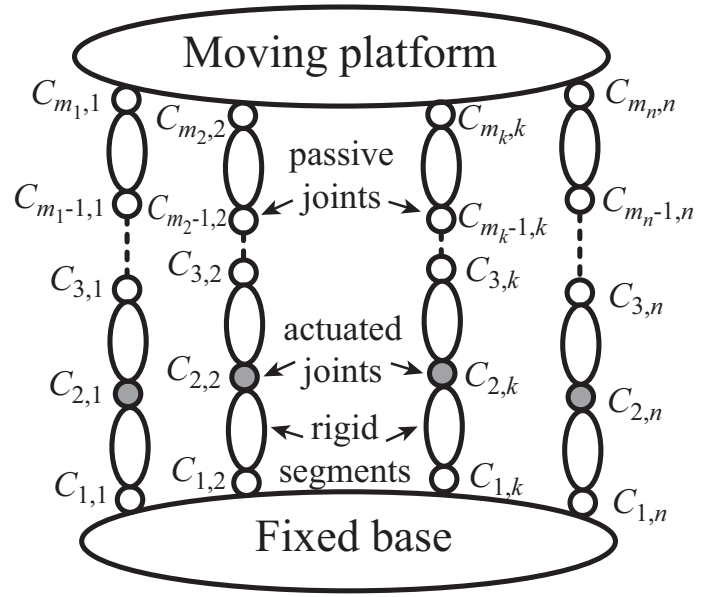

(a) Kinematic chain $\left(C_{j, k}\right.$ is the joint $j$ of the leg $k$, and $m_{k}$ is the total number of joints for the leg $k)$

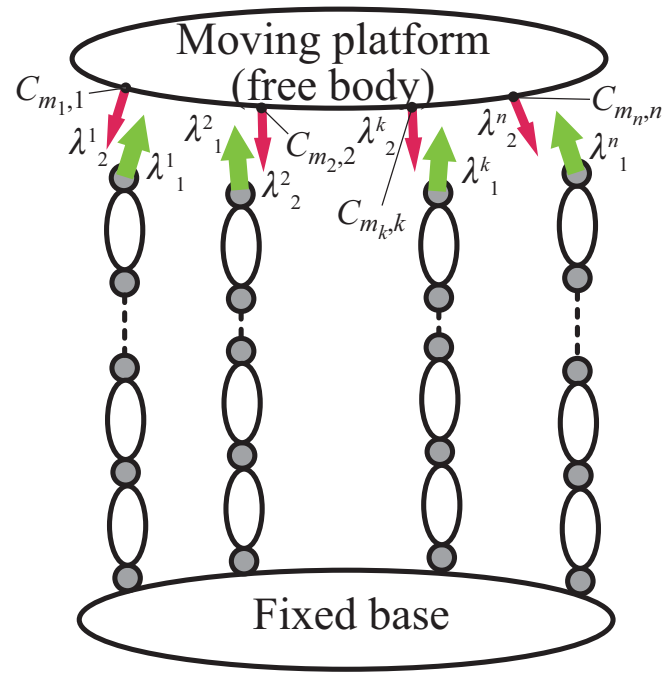

(b) Virtual tree structure:

Figure 2: A general parallel robot (grey circles denote actuated joints).

1. all closed loops are virtually opened to virtually disassemble the platform from the rest of the structure (Fig. 2(b)); each leg joint is virtually considered to be actuated (even for unactuated actual joints) so that the robot becomes a virtual tree structure while the moving platform becomes a virtual free body; the dynamic models of the virtual tree structure and of the virtual free platform are then computed with a systematic procedure based on the NewtonEuler principle, and

2. the loops are then closed using loop-closure equations and Lagrange multipliers (which represent the joint constraints applied to the platform that are required to close the loops of the real robot), which involve the computation of robot Jacobian matrices.

In what follows, the computation of the IDIM of the virtual tree structure and of the platform is recalled, and then a straightforward way to compute the Jacobian matrices required to calculate the closed-loop constraints is detailed.

\section{$2.2 \quad I D I M$ of Tree Open Loop Robots}

According to [14], the complete rigid dynamic model of any open-loop tree structure can be linearly written in terms of a $\left(n_{t} \times 1\right)$ vector with respect to the standard dynamic parameters $\chi_{s t_{t}}\left(n_{t}\right.$ denotes the total number of joints for the virtual tree structure),

$$
\boldsymbol{\tau}_{i d m_{t}}\left(\mathbf{q}_{t}, \dot{\mathbf{q}}_{t}, \ddot{\mathbf{q}}_{t}\right)=\operatorname{IDM}_{s t_{t}}\left(\mathbf{q}_{t}, \dot{\mathbf{q}}_{t}, \ddot{\mathbf{q}}_{t}\right) \chi_{s t_{t}}
$$

where $\boldsymbol{\tau}_{i d m_{t}}$ is the $\left(n_{t} \times 1\right)$ vector of the input efforts of the virtual tree structure, IDM $_{s t_{t}}$ is the $\left(n_{t} \times n_{s t_{t}}\right)$ Jacobian matrix of $\boldsymbol{\tau}_{i d m_{t}}$ with respect to the $\left(n_{s t_{t}} \times 1\right)$ vector $\boldsymbol{\chi}_{s t_{t}}$ of the standard dynamic parameters given by $\boldsymbol{\chi}_{s t_{t}}^{T}=\left[\chi_{s t}^{11 T}, \boldsymbol{\chi}_{s t}^{21 T}, \ldots \chi_{s t}^{m_{n}, n T}\right]$ and $\mathbf{q}_{t}, \dot{\mathbf{q}}_{t}, \ddot{\mathbf{q}}_{t}$ are the vectors of all joint positions, velocities and accelerations of the virtual tree structure robot, respectively.

For rigid robots, the vector $\chi_{s t}^{j k}$ of link $j$ for leg $k$ (denoted in what follows as the link $j k$ ) is composed of 14 standard dynamic parameters such that:

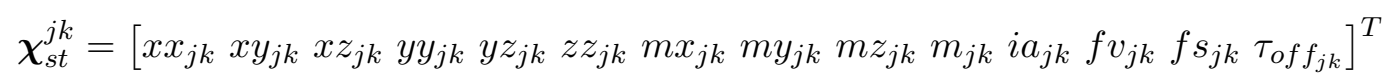

where: 
- $x x_{j k}, x y_{j k}, x z_{j k}, y y_{j k}, y z_{j k}, z z_{j k}$ are the 6 independent components of the inertia matrix $\mathbf{I}_{j k}$ of link $j k$ at the origin of frame $j k$, i.e.

$$
\mathbf{I}_{j k}=\left[\begin{array}{lll}
x x_{j k} & x y_{j k} & x z_{j k} \\
x y_{j k} & y y_{j k} & y z_{j k} \\
x z_{j k} & y z_{j k} & z z_{j k}
\end{array}\right]
$$

- $m_{j k}$ is its mass,

- $m x_{j k}, m y_{j k}, m z_{j k}$ are the 3 components of the first moment of link $j k$, i.e.

$$
m_{j k}{ }^{j k} \overrightarrow{O_{j k} S_{j k}}=\left[\begin{array}{lll}
m x_{j k} & m y_{j k} & m z_{j k}
\end{array}\right]^{T}
$$

where ${ }^{j k} \overrightarrow{O_{j k} S_{j k}}$ is the position of the center of mass of the link $j k$ expressed in the frame attached at the origin of the considered link [14],

- $i a_{j k}$ is the total inertia moment for rotor and gears of the drive train,

- $f v_{j k}, f s_{j k}$ are the viscous and Coulomb friction coefficients in the joint $j k$, respectively, and $\tau_{o f f_{j k}}=\tau_{o f f s_{j k}}+\tau_{o f f \tau_{j k}}$ is an offset parameter which regroups the current amplifier offset $\tau_{o f f \tau_{j k}}$ and the asymmetrical Coulomb friction coefficient $\tau_{o f f s_{j k}}$ such as the friction effort $\tau_{f_{j k}}$ in the joint $j k$ is given by the relation:

$$
\tau_{f_{j k}}=f v_{j k} \dot{q}_{j k}+f s_{j k} \operatorname{sign}\left(\dot{q}_{j k}\right)+\tau_{o f f_{j k}}
$$

where $\dot{q}_{j k}$ is the joint $j k$ generalized velocity.

In the same way, the IDIM of the platform can be obtained as:

$$
\tau_{p}(\mathbf{x}, \mathbf{t}, \dot{\mathbf{t}})=\operatorname{IDM}_{p}(\mathbf{x}, \mathbf{t}, \dot{\mathbf{t}}) \chi_{p}
$$

where $\boldsymbol{\tau}_{p}$ is the $(6 \times 1)$ vector of platform reaction wrench, $\mathbf{I D M}_{p}$ is the $(6 \times 10)$ Jacobian matrix of $\boldsymbol{\tau}_{p}$, with respect to the $(10 \times 1)$ vector $\boldsymbol{\chi}_{p}$ of the platform inertial standard parameters ${ }^{2}$ and $\mathbf{x}, \mathbf{t}, \dot{\mathbf{t}}$ are the platform pose, twist and acceleration quantities, respectively.

Various methods can be used to systematically derive these equations. Here, an algorithm based on the use of the modified Denavit-Hartenberg robot geometric description and the Newton-Euler principle is applied. This modeling is known to give the dynamic model equations in the most compact form [14].

\subsection{IDIM of Parallel Robots with no Actuation Redundancy}

The IDIM of the virtual tree structure and of the free moving platform does not take into account the closed loop characteristics of parallel robots: among all joint and platform coordinates $\mathbf{q}_{t}$ and $\mathbf{x}$ of the virtual robot (Fig. 2(b)), only a subset denoted as $\mathbf{q}$ is independent in the real robot (actual actuated joints positions are indeed a subset of $\mathbf{q}_{t}$ ). All these variables are linked through the loop-closure equations of the real robot that can be obtained by expressing the (translational and rotational) displacement $\mathbf{x}_{k}$ of the last joints of each leg located at $C_{m_{k}, k}$ (that belong to both platform and leg $k$-Fig. 2) in two different ways: (i) as a function of the platform coordinates $\mathbf{x}$ and (ii) as a function of all joint coordinates $\mathbf{q}_{t}$ (also corresponding to the joint coordinates of the virtual tree structure), such that:

$$
\mathbf{f}\left(\mathbf{x}, \mathbf{q}_{t}\right)=\left[\begin{array}{c}
\mathbf{x}_{1}(\mathbf{x})-\mathbf{x}_{1}\left(\mathbf{q}_{t}\right) \\
\vdots \\
\mathbf{x}_{n}(\mathbf{x})-\mathbf{x}_{n}\left(\mathbf{q}_{t}\right)
\end{array}\right]=\mathbf{0}
$$

\footnotetext{
${ }^{2}$ The number of standard parameters of a free rigid body can be reduced to 10 inertial parameters as it is not necessary to consider the parameters $i a_{j}, f v_{j}, f s_{j}$ and $\tau_{o f f_{j}}$ related to actuated joint drive chains.
} 
The main problem with (7) is that it is usually difficult to straightworwardly solve these equations. Therefore, it is better to express the reduced loop-closure equations of the parallel robot which are known to be simpler to obtain [1] and that directly relate the displacements $\mathbf{q}$ of the actuated joints to the moving platform independent coordinates $\mathbf{x}_{\text {ind }}$ (defined as a subset of $\mathbf{x}$ ):

$$
\mathbf{f}_{p}\left(\mathbf{x}_{\text {ind }}, \mathbf{q}\right)=\mathbf{0}
$$

and to solve then the reduced forward kinematic problem $(f k p)$ which gives $\mathbf{x}_{\text {ind }}$ as a function of $\mathbf{q}$. Obviously, this problem can be also tedious, but:

- the equations (8) are simpler to solve than the equations (7),

- if the problem cannot be solved analytically, a numeric procedure may be applied [1].

Once the values of $\mathbf{x}_{i n d}$ are found as a function of $\mathbf{q}$, it is possible to solve the inverse kinematic problem using (7) in order to express all joint coordinates as a function of $\mathbf{x}$ (that is function of $\mathbf{x}_{\text {ind }}$ ), and thus of $\mathbf{q}$. This problem is generally easy for usual parallel robots [1] and, even for more complicated cases, can now be solved using advanced mathematical methods [24].

Differentiating (7) and (8) with respect to time, the following expressions can be obtained:

$$
\begin{aligned}
& \mathbf{J}_{t k} \mathbf{v}-\mathbf{J}_{k} \dot{\mathbf{q}}_{t}=\mathbf{0} \Rightarrow \dot{\mathbf{q}}_{t}=\mathbf{J}_{k}^{-1} \mathbf{J}_{t k} \mathbf{v}, \\
& \mathbf{J}_{t k} \dot{\mathbf{v}}+\dot{\mathbf{J}}_{t k} \mathbf{v}-\mathbf{J}_{k} \ddot{\mathbf{q}} t-\dot{\mathbf{J}}_{k} \dot{\mathbf{q}}_{t}=\mathbf{0} \\
& \Rightarrow \ddot{\mathbf{q}}_{t}=\mathbf{J}_{k}^{-1}\left(\mathbf{J}_{t k} \dot{\mathbf{v}}+\dot{\mathbf{J}}_{t k} \mathbf{v}-\dot{\mathbf{J}}_{k} \dot{\mathbf{q}}_{t}\right)
\end{aligned}
$$

and

$$
\begin{gathered}
\mathbf{A}_{p} \mathbf{v}+\mathbf{B}_{p} \dot{\mathbf{q}}=\mathbf{0} \Rightarrow \mathbf{v}=-\mathbf{A}_{p}^{-1} \mathbf{B}_{p} \dot{\mathbf{q}}=\mathbf{J}_{p} \dot{\mathbf{q}} \\
\mathbf{A}_{p} \dot{\mathbf{v}}+\dot{\mathbf{A}}_{p} \mathbf{v}+\mathbf{B}_{p} \ddot{\mathbf{q}}+\dot{\mathbf{B}}_{p} \dot{\mathbf{q}}=\mathbf{0} \\
\Rightarrow \dot{\mathbf{v}}=-\mathbf{A}_{p}^{-1}\left(\dot{\mathbf{A}}_{p} \mathbf{v}+\mathbf{B}_{p} \ddot{\mathbf{q}}+\dot{\mathbf{B}}_{p} \dot{\mathbf{q}}\right)
\end{gathered}
$$

where

$$
\begin{aligned}
\mathbf{A}_{p} & =\left[\frac{\partial \mathbf{f}_{p}}{\partial \mathbf{x}_{i n d}}\right] \mathbf{T}, \mathbf{B}_{p}=\left[\frac{\partial \mathbf{f}_{p}}{\partial \mathbf{q}}\right] \\
\mathbf{J}_{t k} & =\left[\frac{\partial \mathbf{f}^{\prime}}{\partial \mathbf{x}_{\text {ind }}}\right], \mathbf{J}_{k}=-\left[\frac{\partial \mathbf{f}^{\prime}}{\partial \mathbf{q}_{t}}\right]
\end{aligned}
$$

with $\mathbf{f}^{\prime}$ a subset of $n_{t}$ independent equations in $\mathbf{f}\left(n_{t}=\sum_{i=1}^{n} m_{i}\right)$, $\mathbf{T}$ a transformation matrix between the platform twist $\mathbf{t}$ and the derivatives with respect to time of the terms $\mathbf{x}_{\text {ind }}$ [1], and $\mathbf{v}$ a vector of the independent coordinates in the platform twist $\mathbf{t}\left(\operatorname{dim} \mathbf{v}=\operatorname{dim} \mathbf{x}_{i n d}=n \leq 6\right)$, defined such that

$$
\mathbf{t}=\mathbf{D v}
$$

In the case of robots with 6 dof, $\mathbf{D}$ is the identity matrix. In these expressions, it should be noted that

- the matrix $\mathbf{J}_{k}$ stacks all Jacobian matrices corresponding to the independent motions of the last joint due to the joint displacements of each serial leg and is thus a square matrix of dimension $\left(n_{t} \times n_{t}\right)$,

- the matrix $\mathbf{J}_{t k}$ is a matrix of dimension $\left(n_{t} \times n\right)$ that can be obtained by considering the rigid body displacement of any point of the robot platform as a function of the platform twist, and

- in the case of parallel robots without actuation redundancy, the matrices $\mathbf{A}_{p}$ and $\mathbf{B}_{p}$ are square of dimension $(n \times n)$. 
Finally, by introducing (11) and (12) into (9) and (10), the expressions of $\dot{\mathbf{q}}_{t}$ and $\ddot{\mathbf{q}}_{t}$ as functions of $\mathbf{q}, \dot{\mathbf{q}}$ and $\ddot{\mathbf{q}}$ can be obtained.

It should be mentioned that all the previous expressions are valuable as long as the robot does not meet any singularity and as long as there is the same number of actuators as the number of platform dof to control. The singularity avoidance or crossing is not the main topic of this paper, and the reader should refer to $[25,26]$ for further developments. In the following of the Section 2.3, it is considered that all these matrices are regular.

To take into account the loop-closure constraints into the dynamic model of the parallel robot, Lagrange multipliers $\boldsymbol{\lambda}^{T}=\left[\begin{array}{ll}\boldsymbol{\lambda}_{1}^{T} & \boldsymbol{\lambda}_{2}^{T}\end{array}\right]$ can be used [14] to compute the $(n \times 1)$ vector of the actuated joint force/torque $\boldsymbol{\tau}_{i d m}$ of the closed-loop structure. $\boldsymbol{\tau}_{i d m}$ can be obtained in relation of the Lagrange multipliers $\boldsymbol{\lambda}$ by

$$
\boldsymbol{\tau}_{i d m}=\left[\mathbf{0},-\mathbf{B}_{p}^{T}\right]\left[\begin{array}{c}
\boldsymbol{\lambda}_{1} \\
\boldsymbol{\lambda}_{2}
\end{array}\right]=-\mathbf{B}^{T} \boldsymbol{\lambda},
$$

where $\boldsymbol{\lambda}$ is calculated from the relation:

$$
\left[\begin{array}{cc}
\mathbf{J}_{k}^{T} & \mathbf{0} \\
-\mathbf{J}_{t k}^{T} & \mathbf{A}_{p}^{T}
\end{array}\right]\left[\begin{array}{c}
\boldsymbol{\lambda}_{1} \\
\boldsymbol{\lambda}_{2}
\end{array}\right]=\mathbf{A}^{T} \boldsymbol{\lambda}=\left[\begin{array}{c}
\boldsymbol{\tau}_{i d m_{t}} \\
\boldsymbol{\tau}_{p r}
\end{array}\right]
$$

In these expressions,

- $\boldsymbol{\lambda}_{1}$ stacks the wrenches $\boldsymbol{\lambda}_{1}^{1}$ to $\boldsymbol{\lambda}_{1}^{n}$ (Fig. 2(b)) applied by the virtual tree structure on the platform at points $C_{m_{k}, k}$, so that that virtual structure can have the same motion as the real parallel robot,

- $\boldsymbol{\lambda}_{2}$ stacks the values of the norms of the wrenches $\boldsymbol{\lambda}_{2}^{1}$ to $\boldsymbol{\lambda}_{2}^{n}$ (Fig. 2(b)) due to the platform dynamics in the platform joints located at $C_{m_{k}, k}$,

- $\mathbf{A}$ is a square matrix of dimension $\left(\left(n_{t}+n\right) \times\left(n_{t}+n\right)\right)$, and

- $\boldsymbol{\tau}_{p r}$ is defined by

$$
\boldsymbol{\tau}_{p r}=\mathbf{D}^{T} \boldsymbol{\tau}_{p}
$$

where $\boldsymbol{\tau}_{p}$ is given in (6) and $\boldsymbol{\tau}_{p r}$ is a subset of forces/moments in $\boldsymbol{\tau}_{p}$ that can be found through the use of the principle of virtual powers, which states that:

$$
\mathbf{v}^{* T} \boldsymbol{\tau}_{p r}=\mathbf{t}^{* T} \boldsymbol{\tau}_{p}=\mathbf{v}^{* T} \mathbf{D}^{T} \boldsymbol{\tau}_{p}
$$

In this equation, the superscript "** stands for a virtual velocity.

Thus, the second equation of the system (16) represents the platform equilibrium so that the loops of the parallel robot can be closed.

Solving (15) and (16), it can be demonstrated that:

$$
\begin{aligned}
\boldsymbol{\tau}_{i d m} & =\mathbf{J}_{t}^{T} \boldsymbol{\tau}_{i d m_{t}}+\mathbf{J}_{p}^{T} \mathbf{D}^{T} \boldsymbol{\tau}_{p} \\
& =\mathbf{J}_{t}^{T} \mathbf{I D M}_{s t_{t}} \boldsymbol{\chi}_{s t_{t}}+\mathbf{J}_{p}^{T} \mathbf{D}^{T} \mathbf{I D M}_{p} \boldsymbol{\chi}_{p} \\
& =\left[\begin{array}{ll}
\mathbf{J}_{t}^{T} \mathbf{I D M}_{s t_{t}} & \mathbf{J}_{p}^{T} \mathbf{D}^{T} \mathbf{I D M}_{p}
\end{array}\right]\left[\begin{array}{ll}
\boldsymbol{\chi}_{s t_{t}}^{T} & \boldsymbol{\chi}_{p}^{T}
\end{array}\right]^{T} \\
& =\mathbf{I D M}_{s t}(\mathbf{q}, \dot{\mathbf{q}}, \ddot{\mathbf{q}}) \boldsymbol{\chi}_{s t}
\end{aligned}
$$

where $\mathbf{J}_{t}=\mathbf{J}_{k}^{-1} \mathbf{J}_{t k} \mathbf{J}_{p}$.

(19) represents the IDIM of the parallel robot without actuation redundancy. 


\section{Inverse Dynamic Identification Model of Parallel Robots with Actuation Redundancy}

\subsection{Inverse Dynamic Model}

Let us consider in this part an actuation redundant parallel robot with $r$ independent dof controlled by $n$ actuators, where $r<n$. Differentiating (7) with respect to time, it can now be proven that the matrix $\mathbf{A}_{p}$ of (11) becomes rectangular of dimension $(n \times r)$, while the matrix $\mathbf{B}_{p}$ stays square of dimension $(n \times n)$ [1].

As a result, the right parts of (11) and (12) must be changed as

$$
\mathbf{A}_{p} \mathbf{v}+\mathbf{B}_{p} \dot{\mathbf{q}}=\mathbf{0} \Rightarrow \dot{\mathbf{q}}=-\mathbf{B}_{p}^{-1} \mathbf{A}_{p} \mathbf{v}=\mathbf{J}_{p}^{i n v} \mathbf{v},
$$

or

$$
\mathbf{v}=\mathbf{J}_{p}^{i n v}+\dot{\mathbf{q}}
$$

where $\mathbf{J}_{p}^{i n v}+$ is the pseudo-inverse of $\mathbf{J}_{p}^{i n v}$ defined by

$$
\mathbf{J}_{p}^{i n v+}=\left(\mathbf{J}_{p}^{i n v T} \mathbf{J}_{p}^{i n v}\right)^{-1} \mathbf{J}_{p}^{i n v T}
$$

and

$$
\begin{aligned}
& \mathbf{A}_{p} \dot{\mathbf{v}}+\dot{\mathbf{A}}_{p} \mathbf{v}+\mathbf{B}_{p} \ddot{\mathbf{q}}+\dot{\mathbf{B}}_{p} \dot{\mathbf{q}}=\mathbf{0} \\
& \Rightarrow \ddot{\mathbf{q}}=-\mathbf{B}_{p}^{-1}\left(\dot{\mathbf{A}}_{p} \mathbf{v}+\mathbf{A}_{p} \dot{\mathbf{v}}+\dot{\mathbf{B}}_{p} \dot{\mathbf{q}}\right)
\end{aligned}
$$

or also

$$
\dot{\mathbf{v}}=-\mathbf{A}_{p}^{+}\left(\dot{\mathbf{A}}_{p} \mathbf{v}+\mathbf{B}_{p} \ddot{\mathbf{q}}+\dot{\mathbf{B}}_{p} \dot{\mathbf{q}}\right)
$$

where $\mathbf{A}_{p}^{+}$is the pseudo-inverse of $\mathbf{A}_{p}$ defined by

$$
\mathbf{A}_{p}^{+}=\left(\mathbf{A}_{p}^{T} \mathbf{A}_{p}\right)^{-1} \mathbf{A}_{p}^{T}
$$

Moreover, the matrix $\mathbf{A}^{T}$ of (16) becomes now a rectangular matrix with $\left(n_{t}+r\right)$ rows and $\left(n_{t}+n\right)$ columns, i.e. the system (16) has more unknowns than equations. Thus, there is an infinity of solutions for the Lagrange multipliers $\boldsymbol{\lambda}$. One solution can be obtained, assuming that the value of the overconstraint $\mathbf{c}$ in the robot is known [5]:

$$
\begin{aligned}
& \boldsymbol{\tau}_{i d m}=\left[\mathbf{0},-\mathbf{B}_{p}^{T}\right] \boldsymbol{\lambda}, \\
& \boldsymbol{\lambda}=\left(\mathbf{A}^{T}\right)^{+}\left[\begin{array}{c}
\boldsymbol{\tau}_{i d m_{t}} \\
\boldsymbol{\tau}_{p r}
\end{array}\right]+\left(\mathbf{I}-\left(\mathbf{A}^{T}\right)^{+} \mathbf{A}^{T}\right) \mathbf{c}
\end{aligned}
$$

where $\left(\mathbf{A}^{T}\right)^{+}$is the Moore-Penrose pseudo-inverse of $\mathbf{A}^{T}$ :

$$
\left(\mathbf{A}^{T}\right)^{+}=\mathbf{A}\left(\mathbf{A}^{T} \mathbf{A}\right)^{-1}
$$

that may be obtained through $S V D$. It should be mentioned that, in most of the cases, the value of $\mathbf{c}$ is set to zero in the controller and, as a result, the vector $\boldsymbol{\lambda}$ is of minimal norm. Obviously, this is not always the case and another (constant or varying) value can be given in order to improve the robot properties, such as increasing the acceleration or payload capacities [4] or even decreasing the backlash [5].

However, as the value of the overcontraint $\mathbf{c}$ is generally not given by industrial controllers, it cannot be set a priori. Thus, the dynamic model (26) cannot be used as it is. We propose here two ways for modifying the inverse dynamic model so that it is possible to obtain a unique IDIM for parallel robot with actuation redundancy. It should be mentioned that these formulations are universal (i.e. they can be obtained for any actuation redundant parallel robot for which the value of the overconstraint is known or not). 


\subsubsection{Case 1: Projection of the input force/torque on the platform}

As matrices $\mathbf{B}_{p}$ and $\mathbf{J}_{k}$ of (11) and (9) are still square and not singular outside of serial singularities [1], Eqs. (15) and (16) can be rewritten as:

$$
\left[\begin{array}{c}
\boldsymbol{\tau}_{i d m} \\
\boldsymbol{\tau}_{i d m_{t}}
\end{array}\right]=\left[\begin{array}{cc}
\mathbf{0} & -\mathbf{B}_{p}^{T} \\
\mathbf{J}_{k}^{T} & \mathbf{0}
\end{array}\right] \boldsymbol{\lambda}, \quad\left[\begin{array}{ll}
-\mathbf{J}_{t k}^{T} & \mathbf{A}_{p}^{T}
\end{array}\right] \boldsymbol{\lambda}=\boldsymbol{\tau}_{p r}
$$

resulting in

$$
\begin{aligned}
\boldsymbol{\tau}_{p r} & =\left[\begin{array}{ll}
-\mathbf{J}_{t k}^{T} & \mathbf{A}_{p}^{T}
\end{array}\right]\left[\begin{array}{cc}
\mathbf{0} & \mathbf{J}_{k}^{-T} \\
-\mathbf{B}_{p}^{-T} & \mathbf{0}
\end{array}\right]\left[\begin{array}{c}
\boldsymbol{\tau}_{i d m} \\
\boldsymbol{\tau}_{i d m_{t}}
\end{array}\right] \\
& =\left[\begin{array}{ll}
-\mathbf{A}_{p}^{T} \mathbf{B}_{p}^{-T} & -\mathbf{J}_{t k}^{T} \mathbf{J}_{k}^{-T}
\end{array}\right]\left[\begin{array}{c}
\boldsymbol{\tau}_{i d m} \\
\boldsymbol{\tau}_{i_{d m}}
\end{array}\right] \\
& =\left[\begin{array}{ll}
\mathbf{J}_{p}^{i n v} T & -\mathbf{J}_{t k}^{T} \mathbf{J}_{k}^{-T}
\end{array}\right]\left[\begin{array}{c}
\boldsymbol{\tau}_{i d m} \\
\boldsymbol{\tau}_{i d m_{t}}
\end{array}\right]
\end{aligned}
$$

with $\mathbf{J}_{p}^{i n v}=-\mathbf{B}_{p}^{-1} \mathbf{A}_{p}$ according to (20).

Introducing (1), (6) and (17) into (29) leads to:

$$
\begin{aligned}
\boldsymbol{\Gamma}_{i d m}^{1}:=\mathbf{J}_{p}^{i n v}{ }^{T} \boldsymbol{\tau}_{i d m} & =\mathbf{J}_{t k}^{T} \mathbf{J}_{k}^{-T} \boldsymbol{\tau}_{i d m_{t}}+\mathbf{D}^{T} \boldsymbol{\tau}_{p} \\
& =\mathbf{J}_{t k}^{T} \mathbf{J}_{k}^{-T} \mathbf{I D M}_{s t_{t}} \boldsymbol{\chi}_{s t_{t}}+\mathbf{D}^{T} \mathbf{I D M}_{p} \boldsymbol{\chi}_{p} \\
& =\left[\begin{array}{ll}
\mathbf{J}_{t k}^{T} \mathbf{J}_{k}^{-T} \mathbf{I D M} \\
\mathbf{I D t}_{t} & \mathbf{D}^{T} \mathbf{I D M}_{p}
\end{array}\right]\left[\begin{array}{ll}
\boldsymbol{\chi}_{s t_{t}}^{T} & \boldsymbol{\chi}_{p}^{T}
\end{array}\right]^{T} \\
& =\mathbf{I D M}_{s t_{1}}^{\text {red }}(\mathbf{q}, \dot{\mathbf{q}}, \ddot{\mathbf{q}}) \boldsymbol{\chi}_{s t}
\end{aligned}
$$

Thus, by projecting the input forces/torques on the platform through the use of the matrix $\mathbf{J}_{p}^{i n v}{ }^{T}$, the inverse dynamic model becomes unique and can be used for identification purpose.

\subsubsection{Case 2: Projection of some input force/torque on actuated bodies}

Another way to compute a unique solution for the inverse dynamic model is the following. The idea is to fulfill the system of equations (16) with $n-r$ equations coming from (15). Let us partition (15) as follows:

$$
\left[\begin{array}{c}
\boldsymbol{\tau}_{i d m}^{1: r} \\
\boldsymbol{\tau}_{i d m}^{r+1: n}
\end{array}\right]=\left[\begin{array}{cc}
\mathbf{0} & -\mathbf{B}_{p}^{1: r^{T}} \\
\mathbf{0} & -\mathbf{B}_{p}^{r+1: n^{T}}
\end{array}\right] \boldsymbol{\lambda}
$$

where $\boldsymbol{\tau}_{i d m}^{1: r}$ and $\boldsymbol{\tau}_{i d m}^{r+1: n}$ are subsets of $\boldsymbol{\tau}_{i d m}\left(\boldsymbol{\tau}_{i d m}^{1: r}\right.$ is of length $r$, and $\boldsymbol{\tau}_{i d m}^{r+1: n}$ is of length $\left.n-r\right)$, and $\mathbf{B}_{p}^{1: r}$ and $\mathbf{B}_{p}^{r+1: n}$ are subsets of $\mathbf{B}_{p}\left(\mathbf{B}_{p}^{1: r}\right.$ is of dimension $r \times n$, and $\mathbf{B}_{p}^{r+1: n}$ of dimension $\left.(n-r) \times n\right)$.

The way of partitioning (15) is obviously not unique. If the robot has got identical legs with symmetrical arrangement (this is the case of the prototype studied in Section 5.1), the $n-r$ equations can be those corresponding to the $n-r$ last actuators. If it is not the case, a study must be completed to find the partitioning that leads to the best identification results (in terms of quality of parameter estimation and input/force torque reconstruction). However, this study is not the topic of the present paper.

Finally, the $n-r$ last equations of (31) can be added into (16) such that the system (15)-(16) becomes:

$$
\begin{aligned}
\boldsymbol{\tau}_{i d m}^{1: r} & =\left[\begin{array}{ll}
\mathbf{0} & -\mathbf{B}_{p}^{1: r}
\end{array}\right] \boldsymbol{\lambda}=\mathbf{B}_{p}^{* T} \boldsymbol{\lambda} \\
{\left[\begin{array}{c}
\boldsymbol{\tau}_{i d m}^{r+1: n} \\
\boldsymbol{\tau}_{i d m_{t}} \\
\boldsymbol{\tau}_{p r}
\end{array}\right] } & =\left[\begin{array}{cc}
\mathbf{0} & -\mathbf{B}_{p}^{1: r^{T}} \\
\mathbf{J}_{k}^{T} & \mathbf{0} \\
-\mathbf{J}_{t k}^{T} & \mathbf{A}_{p}^{T}
\end{array}\right] \boldsymbol{\lambda}=\mathbf{A}_{p}^{* T} \boldsymbol{\lambda}
\end{aligned}
$$


Solving (33), it comes that:

$$
\boldsymbol{\lambda}=\mathbf{A}_{p}^{*-T}\left[\begin{array}{c}
\boldsymbol{\tau}_{i d m}^{r+1: n} \\
\boldsymbol{\tau}_{i d m_{t}} \\
\boldsymbol{\tau}_{p r}
\end{array}\right]=\mathbf{A}_{p_{1}} \boldsymbol{\tau}_{i d m}^{r+1: n}+\mathbf{A}_{p_{2}} \boldsymbol{\tau}_{i d m_{t}}+\mathbf{A}_{p_{3}} \boldsymbol{\tau}_{p r}
$$

where $\mathbf{A}_{p}^{*-T}=\left[\begin{array}{lll}\mathbf{A}_{p_{1}} & \mathbf{A}_{p_{2}} & \mathbf{A}_{p_{3}}\end{array}\right]$. It should be mentioned that a sufficient condition for $\mathbf{A}_{p}^{* T}$ to be invertible is that matrices $\mathbf{J}_{k}^{T}, \mathbf{J}_{t k}^{T}, \mathbf{B}_{p}^{1: r^{T}}$ and $\mathbf{A}_{p}^{T}$ are of full rank, i.e. the robot does not cross any serial or parallel singularities [1].

Introducing (33) into (32) leads to:

$$
\begin{aligned}
\boldsymbol{\tau}_{i d m}^{1: r} & =\mathbf{B}_{p}^{* T}\left(\mathbf{A}_{p_{1}} \boldsymbol{\tau}_{i d m}^{r+1: n}+\mathbf{A}_{p_{2}} \boldsymbol{\tau}_{i d m_{t}}+\mathbf{A}_{p_{3}} \boldsymbol{\tau}_{p r}\right) \\
& =\mathbf{B}_{p}^{* T} \mathbf{A}_{p_{1}}^{i n v} \boldsymbol{\tau}_{i d m}^{r+1: n}+\mathbf{B}_{p}^{* T} \mathbf{A}_{p_{2}} \boldsymbol{\tau}_{i d m_{t}}+\mathbf{B}_{p}^{* T} \mathbf{A}_{p_{3}} \boldsymbol{\tau}_{p r} \\
& =\mathbf{J}_{\boldsymbol{\tau}_{r+1: n}}^{T} \boldsymbol{\tau}_{i d m}^{r+1: n}+\mathbf{J}_{\boldsymbol{\tau}_{i d m}}^{T} \boldsymbol{\tau}_{i d m_{t}}+\mathbf{J}_{\boldsymbol{\tau}_{p r}}^{T} \boldsymbol{\tau}_{p r}
\end{aligned}
$$

Introducing (1), (6) and (17) into (35) leads to:

$$
\begin{aligned}
\boldsymbol{\Gamma}_{i d m}^{2} & =\mathbf{J}_{\tau_{i d m}}^{T} \boldsymbol{\tau}_{i d m_{t}}+\mathbf{J}_{\tau_{p r}}^{T} \boldsymbol{\tau}_{p r} \\
& =\mathbf{J}_{\tau_{i d m}}^{T} \mathbf{I D M}_{s t_{t}} \boldsymbol{\chi}_{s t_{t}}+\mathbf{J}_{\tau_{p r}}^{T} \mathbf{D}^{T} \mathbf{I D M}_{p} \boldsymbol{\chi}_{p} \\
& =\left[\begin{array}{lll}
\mathbf{J}_{\boldsymbol{\tau}_{\text {idm }}}^{T} \mathbf{I D M}_{s t_{t}} & \mathbf{J}_{\tau_{p r}}^{T} \mathbf{D}^{T} \mathbf{I D M}_{p}
\end{array}\right]\left[\begin{array}{ll}
\boldsymbol{\chi}_{s t_{t}}^{T} & \boldsymbol{\chi}_{p}^{T}
\end{array}\right]^{T} \\
& =\mathbf{I D M}_{\text {st }_{2}}^{r(\mathbf{q}, \dot{\mathbf{q}}, \ddot{\mathbf{q}}) \boldsymbol{\chi}_{s t}}
\end{aligned}
$$

where $\boldsymbol{\Gamma}_{i d m}^{2}:=\boldsymbol{\tau}_{i d m}^{1: r}-\mathbf{J}_{\boldsymbol{\tau}_{r+1: n}}^{T} \boldsymbol{\tau}_{i d m}^{r+1: n}$. Thus, by projecting the input forces/torques of $n-r$ actuators on $r$ other actuators, the inverse dynamic model becomes also unique and can be used for identification purpose.

\subsection{Computation of the IDIM including the payload}

Because of perturbations due to noise measurement and modeling errors, the actual force/torque $\tau$ differs from $\boldsymbol{\tau}_{i d m}$. This involves that the value of $\boldsymbol{\Gamma}^{j}$ calculated using the values of the measured force/torques $\boldsymbol{\tau}$ differs from $\boldsymbol{\Gamma}_{i d m}^{j}$ by an error $\mathbf{e}$, such that

$$
\Gamma^{j}=\Gamma_{i d m}^{j}+\mathbf{e}=\mathbf{I D M}_{s t_{j}}^{r e d} \chi_{s t}+\mathbf{e}
$$

Equation (37) represents the Inverse Dynamic Identification Model (IDIM).

The payload is considered as an additional link (denoted as link $l$ ) fixed to the robot platform [9]. Model (37) then becomes:

$$
\begin{aligned}
\boldsymbol{\Gamma}^{j} & =\left[\begin{array}{ll}
\mathbf{I D M}_{s t_{j}}^{r e d} & \mathbf{I D M}_{l}
\end{array}\right]\left[\begin{array}{c}
\chi_{s t} \\
\chi_{l}
\end{array}\right]+\mathbf{e} \\
& =\mathbf{I D M}_{t o t} \boldsymbol{\chi}_{t o t}+\mathbf{e}
\end{aligned}
$$

where

- $\chi_{l}$ is the $\left(n_{l} \times 1\right)$ vector of the inertial parameters of the payload;

- $\mathbf{I D M}_{l}$ is the $\left(n \times n_{l}\right)$ Jacobian matrix of $\boldsymbol{\Gamma}_{i d m}^{j}$ with respect to the vector $\chi_{l}$. 


\section{Identification Procedure}

\subsection{Computation of the Base Parameters}

In this Section, the off-line identification of the dynamic parameters is considered, given measured or estimated off-line data for $\boldsymbol{\tau}$ and $(\mathbf{q}, \dot{\mathbf{q}}, \ddot{\mathbf{q}})$, collected while the robot is tracking some planned trajectories. The model (37) is sampled at frequency $f_{m}$ in order to get an over-determined linear system of $r_{f_{m}}$ equations and $n_{s t}$ unknowns:

$$
\mathbf{Y}_{f_{m}}(\hat{\mathbf{q}}, \boldsymbol{\tau})=\mathbf{W}_{f_{m}}^{s t}(\hat{\mathbf{q}}, \hat{\dot{\mathbf{q}}}, \hat{\ddot{\mathbf{q}}}) \chi_{s t}+\boldsymbol{\rho}_{f_{m}}
$$

where $(\hat{\mathbf{q}}, \hat{\dot{\mathbf{q}}}, \hat{\overrightarrow{\mathbf{q}}})$ is an estimation of $(\mathbf{q}, \dot{\mathbf{q}}, \ddot{\mathbf{q}})$, respectively, obtained by sampling and band-pass filtering the measure of $\mathbf{q}$ [27], $\boldsymbol{\rho}_{f_{m}}$ is the $\left(r_{f_{m}} \times 1\right)$ vector of errors, $\mathbf{Y}_{f_{m}}$ is the $\left(r_{f_{m}} \times 1\right)$ vector of the inputs $\Gamma^{j}$, sampled at frequency $f_{m}$ and $\mathbf{W}_{f_{m}}^{s t}(\hat{\mathbf{q}}, \hat{\dot{\mathbf{q}}}, \hat{\overrightarrow{\mathbf{q}}})$ is the $\left(r_{f_{m}} \times n_{s t}\right)$ observation matrix.

The forces/torques $\boldsymbol{\tau}$ (and thus the values of $\boldsymbol{\Gamma}^{j}$ ) are perturbed by high frequency unmodeled friction and flexibility force/torque of the joint drive chain which is rejected by the closed loop control. These force/torque ripples are eliminated with a parallel decimation procedure which low pass filters in parallel $\mathbf{Y}_{f_{m}}$ and each column of $\mathbf{W}_{f_{m}}^{\text {st }}$ and re-samples them at a lower rate, keeping one sample over $n_{d}$. This parallel decimation can be carried out with the MATLAB decimate function, where the low pass filter cutoff frequency, $\omega_{f p}=2 \pi 0.8 f_{m} /\left(2 n_{d}\right)$, is chosen in order to keep $\mathbf{Y}_{f_{m}}$ and $\mathbf{W}_{f_{m}}^{s t}$ in the frequency range of the model dynamics. After the data acquisition procedure and the parallel decimation of (39), we obtain an over-determined linear system

$$
\mathbf{Y}(\boldsymbol{\tau})=\mathbf{W}^{s t}(\hat{\mathbf{q}}, \hat{\dot{\mathbf{q}}}, \hat{\ddot{\mathbf{q}}}) \chi_{s t}+\boldsymbol{\rho}
$$

where $\boldsymbol{\rho}$ is the $\left(r_{c} \times 1\right)$ vector of errors, $\mathbf{Y}$ is the $\left(r_{c} \times 1\right)$ vector of the inputs and $\mathbf{W}^{s t}(\hat{\mathbf{q}}, \hat{\dot{\mathbf{q}}}, \hat{\ddot{\mathbf{q}}})$ is the $\left(r_{c} \times n_{s t}\right)$ observation matrix.

It should be noted that no error is introduced by the parallel filtering process in the linear relation (40) compared with (39). In [27], practical rules for tuning this filter are given.

In $\mathbf{Y}$ and $\mathbf{W}^{s t}$, the equations corresponding to the $j$-th line of the vector $\boldsymbol{\Gamma}^{j}$ and of the matrix $\mathbf{I D M}_{s t_{j}}^{\text {red }}$ are sorted in order to regroup the equations of each line altogether such that: $\mathbf{Y}^{T}=$ $\left[\left(\mathbf{Y}^{1}\right)^{T}, \cdots,\left(\mathbf{Y}^{n}\right)^{T}\right], \mathbf{W}^{s t}=\left[\left(\mathbf{W}^{1}\right)^{T}, \cdots,\left(\mathbf{W}^{n}\right)^{T}\right]^{T}$, where $\mathbf{Y}^{j}$ and $\mathbf{W}^{j}$ represent the $r_{c} / n$ equations of the $j$-th line of the vector $\boldsymbol{\Gamma}^{j}$ and of the matrix $\mathbf{I D M}_{s t_{j}}^{r e d}$.

The identifiable parameters are the base parameters which are the minimum number of dynamic parameters from which the dynamic model can be calculated [14]. The minimal dynamic model can be written using the $n_{b}$ base dynamic parameters $\chi$ as follows:

$$
\mathbf{Y}=\mathbf{W}(\mathbf{q}, \dot{\mathbf{q}}, \ddot{\mathbf{q}}) \chi+\rho
$$

where $\mathbf{W}$ is a subset of independent columns in $\mathbf{W}^{\text {st }}$ which defines the identifiable parameters. Several methods exist for the computation of these subsets (analytical [14] or numerical [28]). In this work, it is preferred to use a numerical method based on $Q R$ factorization.

There is infinity of possible subsets of base parameters, as presented in [29, 28]. In [29], the authors test different subsets (obtained via the $S V D$ of the observation matrix [28]) and keep the one which leads to the best conditioning index of the observation matrix. Even if it is computationally efficient, this method has a drawback: for a parallel robot with identical legs, it can lead to a set of base parameters which does not conserve the symmetry properties of the robot legs. Obviously, for avoiding this problem, it can be set a priori that some parameters are equivalent, which involves to sum their corresponding columns in the observation matrix. However, as there can be some small variations in the parameters values due to the manufacturing process, it is worth to avoid this $a$ priori regroupment and check it a posteriori on the identified values.

Here, a method is described that avoids these drawbacks. For presenting it, let us make some brief recalls on the computation of the base parameters via $Q R$ factorization. The $Q R$ factorization 
of the matrix $\mathbf{W}^{\text {st }}$ of (40) takes the form

$$
\mathbf{Q}^{T} \mathbf{W}^{s t}=\left[\begin{array}{c}
\mathbf{R} \\
\mathbf{0}
\end{array}\right]
$$

where $\mathbf{Q}$ is a $(r \times r)$ orthogonal matrix and $\mathbf{R}$ is upper triangular. If the absolute value $\left|R_{k k}\right|$ of the $k$-th component located on the diagonal of $\mathbf{R}$ is inferior to $\alpha$ ( $\alpha$ is the numerical rank - different from 0 because of round-off errors - and can be chosen such that $\alpha=\epsilon \max \left|R_{j j}\right|$, where $\epsilon$ is a small coefficient depending on the level of perturbations in $\mathbf{W}^{s t}$ (due to noise measurement and error modelling) and $\max \left|R_{j j}\right|$ is the largest diagonal absolute value of $\mathbf{R}[28]$ ), the $k$-th column $\mathbf{W}_{k}^{s t}$ of $\mathbf{W}^{s t}$ can be deleted. At the end of the procedure, $\left(n_{s t}-n_{b}\right)$ columns of $\mathbf{W}^{s t}$ have been deleted that correspond to $\left(n_{s t}-n_{b}\right)$ standard parameters removed from vector $\chi_{s t}$ to keep a set of $n_{b}$ base parameters $\chi$.

Because the $Q R$ algorithm starts from the last columns to the first of $\mathbf{W}^{s t}$, the $\left(n_{s t}-n_{b}\right)$ standard parameters to delete are dependent on the ordering of the columns of that matrix. For serial robots, the matrix $\mathbf{W}^{s t}$ is built such that the columns with the smaller indices are those corresponding to the links closest from the base. Thus, using the previous algorithm, the parameters with the smallest influence (those of the wrist) are eliminated from the base parameters.

For parallel robots, to take into account the symmetry in the leg dynamic parameters, it is preferable to order the columns of $\mathbf{W}^{\text {st }}$ such that

$$
\mathbf{W}_{r}^{s t}=\left[\begin{array}{lllll}
\mathbf{W}_{p}^{s t} & \mathbf{W}_{\chi_{1}, 1: n}^{s t} & \mathbf{W}_{\chi_{2}, 1: n}^{s t} & \cdots & \mathbf{W}_{\chi_{n_{s t} t_{l e g}, 1: n}^{s t}}^{s}
\end{array}\right]
$$

where $n_{s t_{l e g}}$ is the number of standard parameters for one leg, matrix $\mathbf{W}_{p}^{s t}$ is the observation matrix corresponding to the platform inertial parameters and matrices $\mathbf{W}_{\chi_{k}, 1: n}^{s t}$ concatenates the columns of matrix $\mathbf{W}^{s t}$ corresponding to the parameters $\chi_{k}$ that are a priori identical for the $n$ legs. Then, $\left(n_{s t}-n_{b}\right)$ columns of $\mathbf{W}_{r}^{s t}$ can be deleted using the previous approach based on the $Q R$ factorization to obtain a new observation matrix $\mathbf{W}$ associated with a set of symmetrical base parameters denoted as $\chi$.

\subsection{Weighted Least Square Identification of the Robot Dynamic Parameters (IDIM-WLS)}

The $L S$ solution $\hat{\chi}$ of (41) is given by

$$
\hat{\boldsymbol{\chi}}=\mathbf{W}^{+} \mathbf{Y} \text { where } \mathbf{W}^{+}=\left(\mathbf{W}^{T} \mathbf{W}\right)^{-1} \mathbf{W}^{T}
$$

is computed using the $Q R$ factorization of $\mathbf{W}$.

Standard deviations $\sigma_{\hat{\chi}_{i}}$ can be estimated assuming that $\mathbf{W}$ is a deterministic matrix and $\boldsymbol{\rho}$ is a zero mean additive independent noise [27] with a covariance matrix $\mathbf{C}_{\boldsymbol{\rho} \rho}$ such that

$$
\mathbf{C}_{\boldsymbol{\rho} \boldsymbol{\rho}}=E\left[\boldsymbol{\rho} \boldsymbol{\rho}^{T}\right]=\sigma_{\boldsymbol{\rho}}^{2} \mathbf{I}_{r_{c}}
$$

$E$ is the expectation operator and $\mathbf{I}_{r_{c}}$, the $\left(r_{c} \times r_{c}\right)$ identity matrix. An unbiased estimation of the standard deviation $\sigma_{\rho}$ is:

$$
\sigma_{\boldsymbol{\rho}}^{2}=\|\mathbf{Y}-\mathbf{W} \hat{\boldsymbol{\chi}}\|^{2} /\left(r_{c}-n_{b}\right)
$$

The covariance matrix of the estimation error is given by:

$$
\mathbf{C}_{\hat{\chi} \hat{\chi}}=E\left[(\chi-\hat{\chi})(\chi-\hat{\chi})^{T}\right]=\sigma_{\rho}^{2}\left(\mathbf{W}^{T} \mathbf{W}\right)^{-1}
$$

$\sigma_{\hat{\chi}_{i}}^{2}=\mathbf{C}_{\hat{\chi} \hat{\chi}}(i, i)$ is the $i$-th diagonal coefficient of $\mathbf{C}_{\hat{\chi} \hat{\chi}}(47)$.

The ordinary $L S$ can be improved by taking into account different standard deviations on actuated joint $j$ equations errors [27]. Data in $\mathbf{Y}$ and $\mathbf{W}$ of (40) are weighted with the inverse of the standard deviation of the error calculated from ordinary $L S$ solution of the equations of joint $j[27]$

$$
\mathbf{Y}^{j}=\mathbf{W}^{j} \chi+\rho^{j}
$$




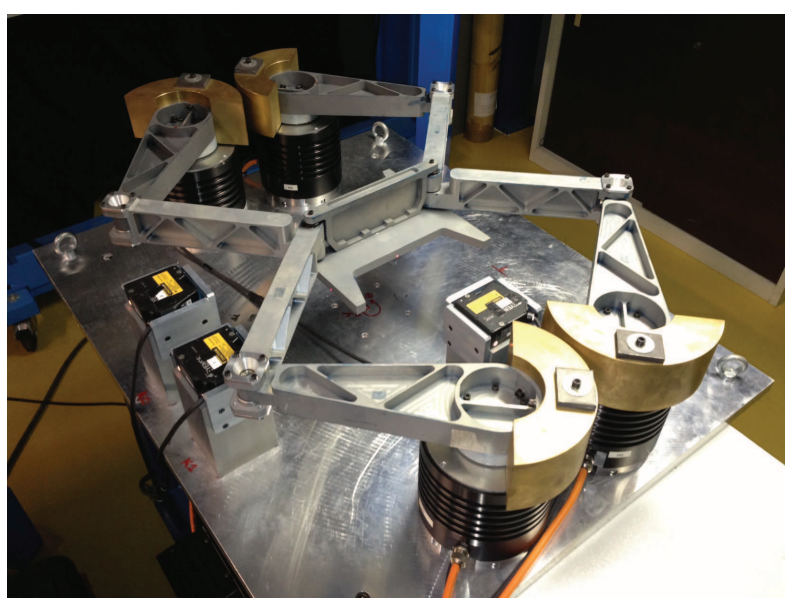

(a) Prototype of DualV robot

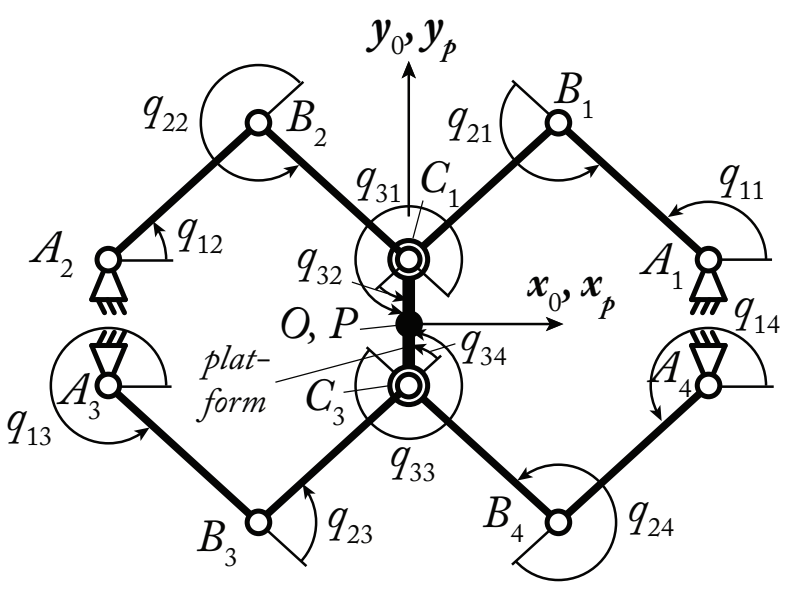

(b) Kinematic description of DualV in a configuration where base frame $x_{0} O y_{0}$ coincides with platform frame $x_{p} P y_{p}$

Figure 3: The DualV.

This weighting operation normalizes the errors in (40) and gives the weighted $L S$ estimation of the parameters (IDIM-WLS).

\subsection{Payload Identification}

In order to identify both the robot and the payload dynamic parameters, using the model (38), it is necessary that the robot carries out two types of trajectories [30]:

1. trajectories without payload, and

2. trajectories with payload fixed to the end-effector.

The sampling and filtering of the model IDIM (38) can be then written as:

$$
\mathbf{Y}=\left[\begin{array}{cc}
\mathbf{W}_{a} & \mathbf{0} \\
\mathbf{W}_{b} & \mathbf{W}_{l}
\end{array}\right]\left[\begin{array}{c}
\chi \\
\chi_{l}
\end{array}\right]+\boldsymbol{\rho}
$$

where

- $\mathbf{W}_{a}$ is the observation matrix of the robot in the unloaded case,

- $\mathbf{W}_{b}$ is the observation matrix of the robot in the loaded case,

- $\mathbf{W}_{l}$ is the observation matrix of the robot corresponding to the payload inertial parameters.

Thus, these two types of trajectories avoid the regrouping of the payload parameters with those of the platform and allow their independent identification. Next section presents experimental results on a prototype of actuation redundant parallel robot.

\section{Case Study}

\subsection{Description of the DualV}

The DualV (Fig. 3) is a prototype of a planar parallel robot with actuation redundancy developed at the LIRMM [21]. This robot has 3 controlled dof (two translations in the plane $(x O y)$ and one rotation about the $z$ axis), but 4 identical legs with one actuator per leg. Thus, its degree of redundancy is equal to 1 . Each leg is composed of one proximal and one distal link. The proximal 


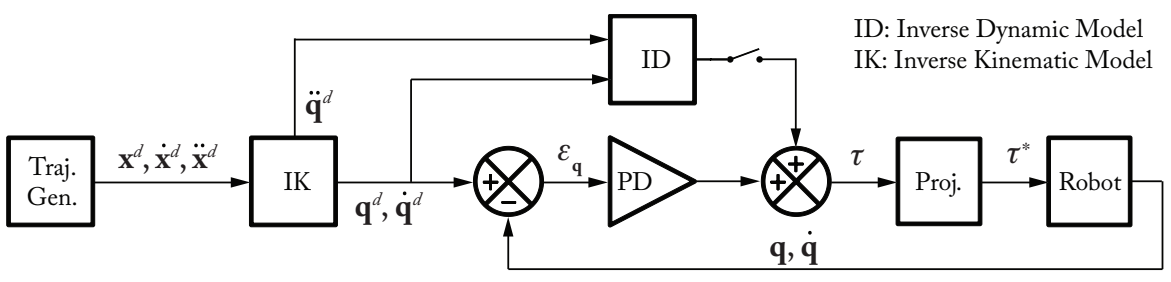

Figure 4: The controller for the DualV.

Table 1: MDH parameters for the frames corresponding to $i$-th robot leg $(i=1, \ldots, 4)$.

\begin{tabular}{lllllll}
\hline \hline$j i$ & $a_{j i}$ & $\mu_{j i}$ & $\gamma_{j i}$ & $d_{j i}$ & $\theta_{j i}$ & $r_{j i}$ \\
\hline $1 i$ & 0 & 1 & $\gamma_{i}$ & $l_{O A_{i}}=0.41 \mathrm{~m}$ & $q_{1 i}-\gamma_{i}$ & 0 \\
$2 i$ & $1 i$ & 0 & 0 & $l_{A_{i} B_{i}}=0.28 \mathrm{~m}$ & $q_{2 i}$ & 0 \\
$3 i$ & $2 i$ & 0 & 0 & $l_{B_{i} C_{i}}=0.28 \mathrm{~m}$ & $q_{3 i}$ & 0 \\
\hline \hline
\end{tabular}

link $A_{i} B_{i}$ is attached to the base by an actuated revolute joint and to the distal link $B_{i} C_{i}$ by a passive revolute joint. The distal link is also attached to the moving platform by a passive revolute joint.

The geometric parameters of the virtual open-loop tree structure are described in Table 1 using the modified Denavit and Hartenberg notation $(M D H)$ [14] (in this table, $\gamma_{1}=15.52 \mathrm{deg}, \gamma_{2}=164.48 \mathrm{deg}$, $\gamma_{3}=-164.48 \mathrm{deg}$ and $\gamma_{4}=-15.52 \mathrm{deg}$ ). The platform and payload are considered as supplementary bodies, the payload being fixed on the platform. They are respectively numbered as bodies 4 and 5 .

The DualV is actuated by four ETEL RTMB0140-100 direct drive actuators, which can deliver maximal torques of $127 \mathrm{Nm}$. The robot is able to achieve accelerations of $25 \mathrm{G}$ in its workspace. The current amplifier can provide directly the measure of the input torque produced by the actuator.

Its controller is an augmented PD control with a dynamic based feedforward and internal-stress avoidance described in Fig. 4. PD controller gains are computed based on the response of a second order differential equation for the tracking error equations, with a chosen bandwidth whose cut-off frequency is equal to $f_{c}=70 \mathrm{~Hz}\left(\omega_{c}=2 \pi f_{c}\right)$ and a damping factor $\xi=0.7$ to provide the fastest response. The bandwidth is chosen just below the natural frequency of the robot estimated at about $100 \mathrm{~Hz}$ using finite element analysis.

The derivative term is filtered using a first-order low-pass filter whose cut-off frequency is set to $70 \mathrm{~Hz}$ to avoid amplifying noise. On this controller, we can switch on or off feed-forward terms to partially compensate for the dynamics of the system or not. This compensation is based on the inverse dynamics computation.

To get rid of the combination of joint torques that create internal-stress rather than operational forces, a projection along the null space of the robot inverse transposed Jacobian matrix $\mathbf{J}_{p}^{i n v} T^{T}$ is performed:

$$
\boldsymbol{\tau}^{*}=\boldsymbol{\tau}-\sum_{i=1}^{n-r}\left(\mathbf{u}_{2_{i}}^{c T} \boldsymbol{\tau}\right) \mathbf{u}_{2_{i}}^{c}
$$

where $\mathbf{u}_{2_{i}}^{c}(i=1, \ldots, n-r)$ span the null space of $\mathbf{J}_{p}^{i n v} T$ (see [20]). Canceling internal stress (from a kinetostatic point of view) is recommended since it leads to less deformation and impact favorably robot's accuracy. Additionally it ensures having the joint torque vector of the lowest norm, which is good from an energy consumption point of view.

Finally, it should be mentionned here that, for the DualV, the controller is known and the value of the overconstraint could be fixed to zero in the identification procedure. However, this could not be the case if the robot were designed by industrial manufacturers that generally give few details about their technology. 


\subsection{Computation of the Inverse Dynamic Model}

The inverse dynamic model of the DualV can be obtained using the equations given in Sections 2 and 3 but is detailed here for reason of clarity.

\subsubsection{Inverse dynamic model of the virtual tree structure and of the virtual free plat- form}

The inverse dynamic model of the open loop virtual structure can be obtained by noticing that each leg is indeed a planar $3 R$ robot in which the last body is massless. Its inverse dynamic model may be found in [31]:

$$
\begin{aligned}
\tau_{t_{1 i}}= & \left(z z_{1 i}+i a_{1 i}+d_{2}^{2} m_{2 i}\right) \ddot{q}_{1 i}+z z_{2 i}\left(\ddot{q}_{1 i}+\ddot{q}_{2 i}\right) \\
& +d_{2} m x_{2 i}\left(\left(2 \ddot{q}_{1 i}+\ddot{q}_{2 i}\right) \cos q_{2 i}-\dot{q}_{2 i}\left(2 \dot{q}_{1 i}+\dot{q}_{2 i}\right) \sin q_{2 i}\right) \\
& +d_{2} m y_{2 i}\left(\left(2 \ddot{q}_{1 i}+\ddot{q}_{2 i}\right) \sin q_{2 i}+\dot{q}_{2 i}\left(2 \dot{q}_{1 i}+\dot{q}_{2 i}\right) \cos q_{2 i}\right) \\
& +f s_{1 i} \operatorname{sign}\left(\dot{q}_{1 i}\right)+f v_{1 i} \dot{q}_{1 i} \\
\tau_{t_{2 i}}= & z z_{2 i}\left(\ddot{q}_{1 i}+\ddot{q}_{2 i}\right)+d_{2} m x_{2 i}\left(\ddot{q}_{1 i} \cos q_{2 i}+\dot{q}_{1 i}^{2} \sin q_{2 i}\right) \\
& +d_{2} m y_{2 i}\left(\ddot{q}_{1 i} \sin q_{2 i}-\dot{q}_{1 i}^{2} \cos q_{2 i}\right) \\
& +f s_{2 i} \operatorname{sign}\left(\dot{q}_{2 i}\right)+f v_{2 i} \dot{q}_{2 i} \\
\tau_{t_{3 i}}= & f s_{3 i} \operatorname{sign}\left(\dot{q}_{3 i}\right)+f v_{3 i} \dot{q}_{3 i}
\end{aligned}
$$

where

- parameters $z z_{j i}, i a_{j i}, m_{j i}, m x_{j i}, m y_{j i}, f s_{j i}, f v_{j i}$ are defined in Section $2.2(j=1,2,3)$,

- angles $q_{j i}$ and length $d_{2}$ are defined in Table 1 and Fig. $3(j=1,2,3)$,

- $\tau_{t_{1 i}}$ is the torque of the virtual actuator located at point $A_{i}, \tau_{t_{2 i}}$ is the torque of the virtual actuator located at point $B_{i}$, and $\tau_{t_{3 i}}$ is the torque of the virtual actuator located at point $C_{i}$. The vector $\boldsymbol{\tau}_{i d m_{t}}$ of (1) stackes all vectors $\boldsymbol{\tau}_{t_{i}}=\left[\begin{array}{lll}\tau_{t_{1 i}} & \tau_{t_{2 i}} & \tau_{t_{3 i}}\end{array}\right]^{T}$.

The inverse dynamic model of the free body corresponding to the platform (body 4) with the payload (body 5 ) in the virtual system is

$$
\begin{aligned}
\tau_{p_{1}}= & \left(m_{4}+m_{5}\right) \ddot{x}-\left(m x_{4}+m x_{5}\right)\left(\ddot{\phi} \sin \phi+\dot{\phi}^{2} \cos \phi\right) \\
& +\left(m y_{4}+m y_{5}\right)\left(-\ddot{\phi} \cos \phi+\dot{\phi}^{2} \sin \phi\right) \\
\tau_{p_{2}}= & \left(m_{4}+m_{5}\right) \ddot{y}+\left(m x_{4}+m x_{5}\right)\left(\ddot{\phi} \cos \phi-\dot{\phi}^{2} \sin \phi\right) \\
& -\left(m y_{4}+m y_{5}\right)\left(\ddot{\phi} \sin \phi+\dot{\phi}^{2} \cos \phi\right) \\
\tau_{p_{3}}= & \left(z z_{4}+z z_{5}\right) \ddot{\phi}+\left(m x_{4}+m x_{5}\right)(\ddot{y} \cos \phi-\ddot{x} \sin \phi) \\
& -\left(m y_{4}+m y_{5}\right)(\ddot{y} \sin \phi+\ddot{x} \cos \phi)
\end{aligned}
$$

with $\tau_{p_{j}}$ being the $j$-th components of the vector $\boldsymbol{\tau}_{p}$ of $(6) ; x, y$ and $\phi$ are the platform coordinates ( $x$ and $y$ are the position of the platform centre, $\phi$ the platform orientation defined as the angle between the axes $x_{0}$ and $\left.x_{p}\right) ; z z_{j}, m_{j}, m x_{j}, m y_{j}$ are defined in Section $2.2(j=4,5)$.

From (51) and (52), the identification model of the tree structure and of the free platform can easily be obtained.

The way to compute the platform and the virtual tree structure joint coordinates, velocities and accelerations is detailed in the following section. 


\subsubsection{Forward kinematic problem of the real parallel robot}

For the DualV, the loop-closure equations $(7)$ can be written as (for $i=1 \ldots 4$ ):

$$
\begin{aligned}
& 0=x-r_{p} \sin (\phi+k \pi)-x_{A_{i}}-d_{2} \cos q_{1 i}-d_{3} \cos \left(q_{1 i}+q_{2 i}\right) \\
& 0=y+r_{p} \cos (\phi+k \pi)-y_{A_{i}}-d_{2} \sin q_{1 i}-d_{3} \sin \left(q_{1 i}+q_{2 i}\right) \\
& 0=\phi+\bar{k} \pi-q_{1 i}-q_{2 i}-q_{3 i}
\end{aligned}
$$

where $r_{p}$ is the half platform length $\left(r_{p}=l_{C_{1} C_{3}} / 2\right), k=0(\bar{k}=1)$ if $i=1,2, k=1(\bar{k}=0)$ if $i=3,4$, and $x_{A_{i}}$ and $y_{A_{i}}$ are the position coordinates along $\mathbf{x}$ and $\mathbf{y}$ axes for the point $A_{i}$.

From the two first lines of (53), the reduced loop-closure equations (8) that directly relate the displacements of the actuated joints to the moving platform coordinates can be obtained after deleting from these expressions the terms in $\cos q_{2 i}$ or $\sin q_{2 i}$ (for $i=1 \ldots 4$ ):

$$
d_{3}^{2}=\left(x_{C_{i}}-x_{B_{i}}\right)^{2}+\left(y_{C_{i}}-y_{B_{i}}\right)^{2}
$$

where $x_{C_{i}}=x-r_{p} \sin (\phi+k \pi)$ and $y_{C_{i}}=y+r_{p} \cos (\phi+k \pi)$ are the position coordinates of point $C_{i}$, and $x_{B_{i}}=x_{A_{i}}+d_{2} \cos q_{1 i}$ and $y_{B_{i}}=y_{A_{i}}+d_{2} \sin q_{1 i}$ are the position coordinates of point $B_{i}$.

Noticing that the forward geometric problem can be solved by

$$
x=\frac{x_{C_{1}}+x_{C_{3}}}{2}, \quad y=\frac{y_{C_{1}}+y_{C_{3}}}{2}, \quad \phi=\tan ^{-1}\left(-\frac{x_{C_{1}}-x_{C_{3}}}{y_{C_{1}}-y_{C_{3}}}\right)
$$

when finding expressions of $x_{C_{j}}$ and $y_{C_{j}}(j=1,3)$ as functions of $q_{1 i}(i=1 \ldots 4)$. These expressions are quite simple to find as the loops formed by the legs 1 and 2, or the legs 3 and 4, are five-bar linkages:

$$
x_{C_{j}}=f_{j} y_{C_{j}}+k_{j}, \quad y_{C_{j}}=\frac{-p_{j} \pm \sqrt{p_{j}^{2}-4 g_{j} r_{j}}}{2 g_{j}}
$$

where

$$
\begin{aligned}
f_{j} & =-\frac{y_{B_{j+1}}-y_{B_{j}}}{x_{B_{j+1}}-x_{B_{j}}}, \quad g_{j}=f_{j}^{2}+1 \\
k_{j} & =\frac{x_{B_{j}}^{2}+y_{B_{j}}^{2}-y_{B_{j}}^{2}-y_{B_{j+1}}^{2}}{2\left(x_{B_{j+1}}-x_{B_{j}}\right)} \\
p_{j} & =2 f_{j}\left(k_{j}-x_{B_{j}}\right)-2 y_{B_{j}} \\
r_{j} & =x_{B_{j}}^{2}+y_{B_{j}}^{2}-d_{3}^{2}+k_{j}^{2}-2 k_{j} x_{B_{j}}
\end{aligned}
$$

In (56), the sign " \pm " denotes the two robot assembly modes, that are considered to be a priori fixed in the identification process as no parallel singularities are crossed.

Then, it comes easily from (53) that:

$$
q_{2 i}=\tan ^{-1}\left(\frac{y_{C_{i}}-y_{B_{i}}}{x_{C_{i}}-x_{B_{i}}}\right)-q_{1 i}, \quad q_{3 i}=\phi+\bar{k} \pi-q_{1 i}-q_{2 i}
$$

Then, differentiating (53) and (54) with respect to time, and simplifying, the matrices $\mathbf{A}_{p}, \mathbf{B}_{p}$, $\mathbf{J}_{k}$ and $\mathbf{J}_{t k}$ of (13) can be found:

$$
\mathbf{a}_{p}^{i}=d_{3}\left[\begin{array}{lll}
c_{12 i} & s_{12 i} & -\sin (\phi+k \pi) s_{12 i}+\cos (\phi+k \pi) c_{12 i}
\end{array}\right]
$$

where $\mathbf{a}_{p}^{i}$ is the $i$-th line of $\mathbf{A}_{p}, c_{12 i}=\cos \left(q_{1 i}+q_{2 i}\right)$ and $s_{12 i}=\sin \left(q_{1 i}+q_{2 i}\right)$,

$$
b_{p}^{i i}=d_{2} d_{3} \sin q_{2 i}
$$

where $b_{p}^{i i}$ is the $i$-th term of the diagonal matrix $\mathbf{B}_{p}$,

$$
\mathbf{J}_{t k}^{T}=\left[\begin{array}{llll}
\mathbf{J}_{t k}^{1 T} & \mathbf{J}_{t k}^{2 T} & \mathbf{J}_{t k}^{3 T} & \mathbf{J}_{t k}^{4 T}
\end{array}\right]
$$


Table 2: Essential parameters of the DualV.

\begin{tabular}{lllll}
\hline \hline & Case 1 & \multicolumn{3}{l}{ Case 2} \\
Param. & Id. Val. & $\% \sigma_{\hat{\chi}_{r i}}$ & Id. Val. & $\% \sigma_{\hat{\chi}_{r i}}$ \\
\hline$z z_{11 R}$ & $4.45 e-2$ & 1.58 & $4.12 e-2$ & 3.14 \\
$z z_{12 R}$ & $4.81 e-2$ & 1.39 & $4.62 e-2$ & 3.09 \\
$z z_{13 R}$ & $4.81 e-2$ & 1.41 & $4.57 e-2$ & 2.54 \\
$z z_{14 R}$ & $5.07 e-2$ & 1.38 & $5.79 e-2$ & 1.89 \\
$z z_{4}$ & $2.06 e-2$ & 1.37 & $2.05 e-2$ & 2.23 \\
$m_{4}$ & $1.92 e+0$ & 1.08 & $1.96 e+0$ & 1.48 \\
$z z_{5}$ & $1.61 e-2$ & 1.71 & $1.64 e-2$ & 3.04 \\
$m x_{5}$ & $-1.27 e-1$ & 0.76 & $-1.27 e-1$ & 1.51 \\
$m_{5}$ & $5.42 e+0$ & 0.30 & $5.52 e+0$ & 0.29 \\
\hline \hline \multicolumn{7}{l}{ Relative error norm $\|\boldsymbol{\rho}\| /\|\mathbf{Y}\| \Rightarrow$ Case 1: 0.110, Case 2: 0.091}
\end{tabular}

in which, for $i=1 \ldots 4$

$$
\mathbf{J}_{t k}^{i}=\left[\begin{array}{ccc}
1 & 0 & -r_{p} \cos (\phi+k \pi) \\
0 & 1 & -r_{p} \sin (\phi+k \pi) \\
0 & 0 & 1
\end{array}\right]
$$

and $\mathbf{J}_{k}$ is a block-diagonal matrix whose $i$-th diagonal element is

$$
\mathbf{J}_{k}^{i}=\left[\begin{array}{ccc}
-d_{2} \sin q_{1 i}-d_{3} \sin \left(q_{1 i}+q_{2 i}\right) & -d_{3} \sin \left(q_{1 i}+q_{2 i}\right) & 0 \\
d_{2} \cos q_{1 i}+d_{3} \cos \left(q_{1 i}+q_{2 i}\right) & d_{3} \cos \left(q_{1 i}+q_{2 i}\right) & 0 \\
1 & 1 & 1
\end{array}\right]
$$

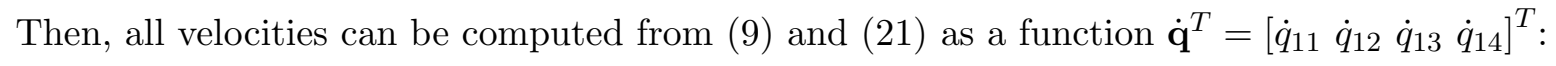

$$
\mathbf{t}=\left[\begin{array}{c}
\dot{x} \\
\dot{y} \\
\dot{\phi}
\end{array}\right]=-\mathbf{A}_{p}^{+} \mathbf{B}_{p} \dot{\mathbf{q}}, \quad \dot{\mathbf{q}}_{i}=\left(\mathbf{J}_{k}^{i}\right)^{-1} \mathbf{J}_{t k}^{i} \mathbf{t}
$$

where $\dot{\mathbf{q}}_{i}$ is the vector of the leg $i$ joint velocities.

Finally, the accelerations can be computed from (10) and (24) using the previous expressions.

Combining these expressions with those of Section 5.2.2 into the equations of Section 3, the identification models of the DualV can be computed.

\subsection{Identification Results}

In this part, experimentations are performed and the dynamic identification model is carried out on the DualV using the modeling approaches presented in Section 3 and the identification procedure proposed in Section 4. To estimate the quality of the identification procedure, a payload mass of $5.37 \mathrm{~kg}$ which has been accurately weighed is mounted of the platform and will be identified in parallel to the robot parameters. Two types of exciting trajectories are then performed, as explained in Section 4.3:

1. trajectories without the payload, and

2. trajectories with the payload fixed to the end-effector.

Before presenting the identification result, it should be noticed that during identification process, some small base parameters remain poorly identifiable because they have no significant contribution in the joint torques. They are canceled to keep a set of essential parameters of a simplified dynamic model with a good accuracy [9]. The essential parameters are calculated using an iterative procedure 

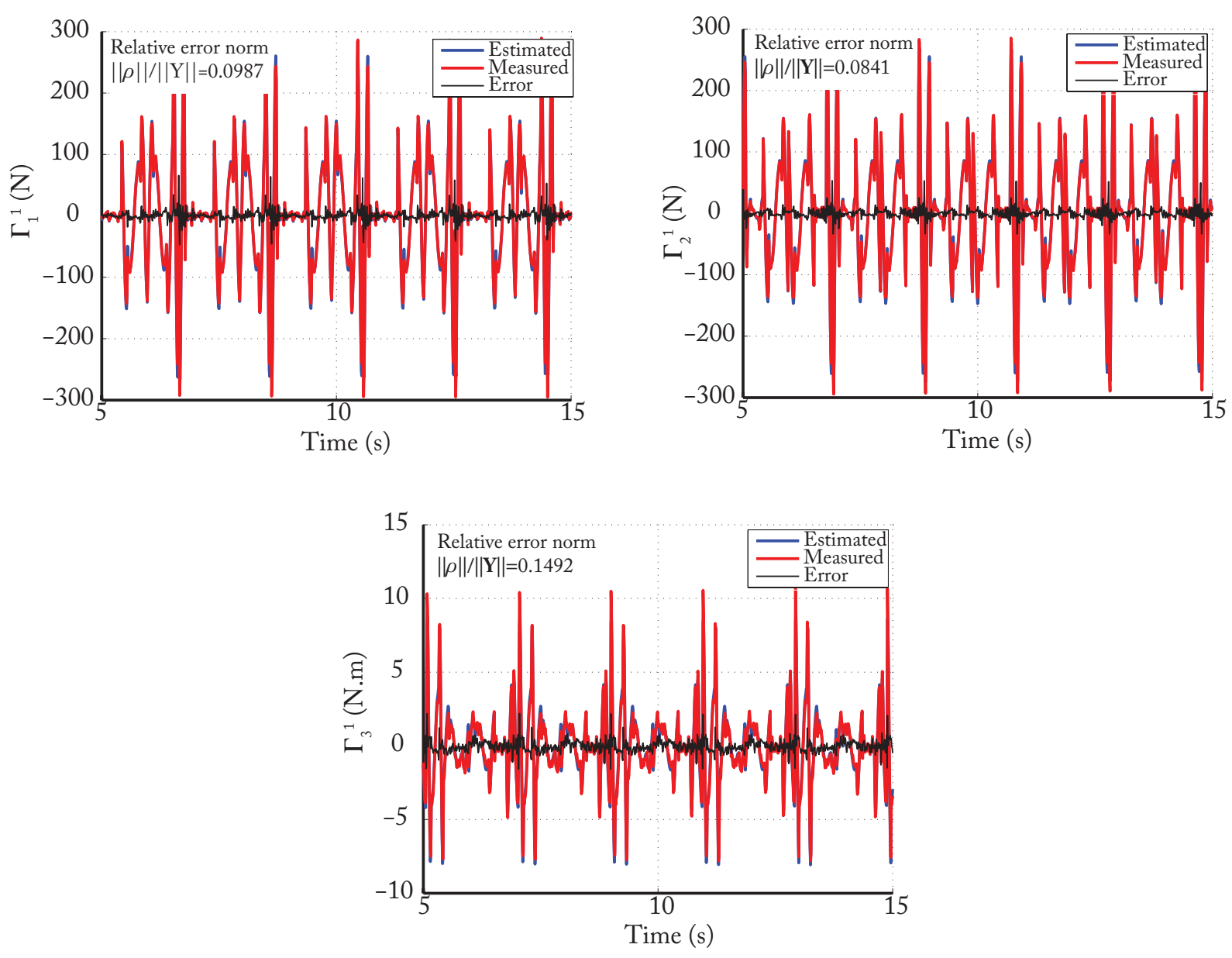

Figure 5: Values of $\boldsymbol{\Gamma}^{1}$, estimated from input torques using the relation $\mathbf{J}_{p}^{\text {inv }}{ }^{T} \boldsymbol{\tau}$ from (30) (red lines) and calculated using identified parameters $\hat{\chi}$ from the relation IDM $\boldsymbol{\chi}$ (blue lines) with the payload of $5.37 \mathrm{~kg}$.

starting from the base parameters estimation. At each step the base parameter which has the largest relative standard deviation $\% \sigma_{\hat{\chi}_{r i}}$ is canceled. A new IDIM-WLS parameter estimation of the simplified model is carried out with new relative error standard deviations $\% \sigma_{\hat{\chi}_{r i}}$. The procedure ends when $\max \left(\% \sigma_{\hat{\chi}_{r i}}\right) / \min \left(\% \sigma_{\hat{\chi}_{r i}}\right)<r_{\sigma}$, where $r_{\sigma}$ is a ratio ideally chosen between 10 and 30 depending on the level of perturbation in $\mathbf{Y}$ and $\mathbf{W}$. In the following of the paper, this ratio is fixed to 10 .

The two proposed identification models are tested:

- Case 1: the IDIM of Section 3.1.1 that projects the input torques on the platform, and

- Case 2: the IDIM of Section 3.1.2 for which the input torque 4 is projected on the actuators 1 to 3 .

Table 2 presents the identification results. Subscript ' $R$ ' stands for the parameters that have been regrouped using the procedure presented in Section 4.1 (here, $z z_{1 i_{R}}=z z_{1 i}+i a_{1 i}+d_{2}^{2} m_{2 i}$ ). It can be observed that the robot parameters have been correctly estimated in both cases. The payload of $5.37 \mathrm{~kg}$ has also been accurately identified. Moreover, the relative error norms in both cases is about 10\%, which shows that the inputs $\boldsymbol{\Gamma}^{j}$ have been correctly reconstructed (see also Figs. 5 and 6).

However, the IDIM of Case 1 leads to lower standard deviations of the identified parameters and to the best payload estimation. 

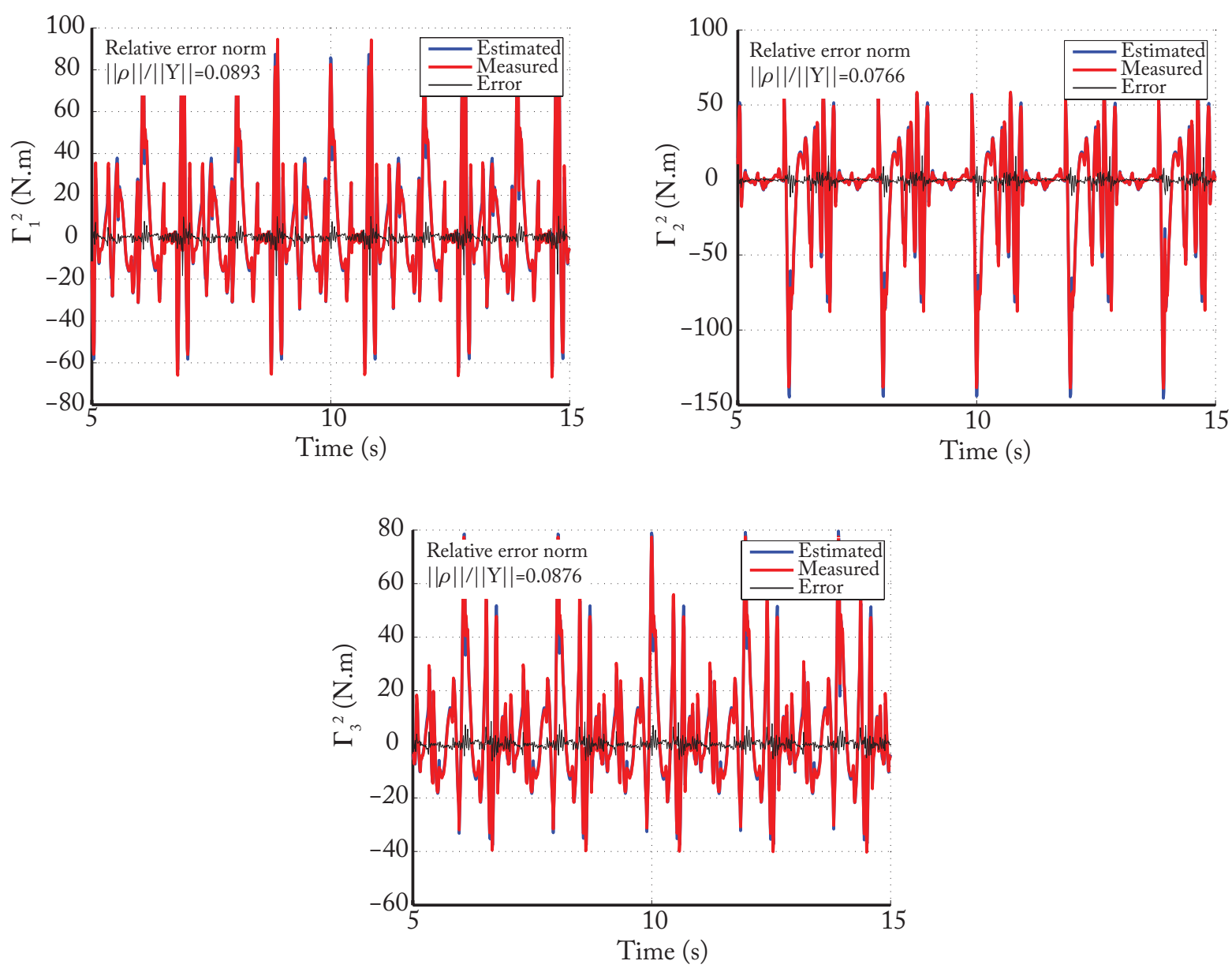

Figure 6: Values of $\boldsymbol{\Gamma}^{2}$, estimated from input torques using the relation $\boldsymbol{\tau}^{1: 3}-\mathbf{J}_{\tau_{4}}^{T} \tau^{4}$ from (36) (red lines) and calculated using identified parameters $\hat{\chi}$ from the relation IDM $\boldsymbol{\chi}$ (blue lines) with the payload of $5.37 \mathrm{~kg}$.

\subsection{Cross-Validations}

To cross-validate the obtained models, the following procedure is used. First, a computed torque control scheme is implemented into the controller. This scheme uses the dynamic models identified into the previous part. Into the controller, the value of the overcontraint is set to zero (Eq. (26)).

Then, two different trajectories are performed and input torques measurements are recorded during these movements. The two trajectories are depicted in Fig. 7. It should be mentioned that, obviously, they are different from the trajectory used for the identification process.

Finally, the measured torques are compared with the calculated torques using (26) by setting the value of the overcontraint equal to zero. The results, in terms of relative error norms, are summarized in the Table 3 (see also Figs. 8 and 9 that show the torques along the two trajectories with the parameters identified in Case 1). It can be seen that the input torques are correctly predicted in both cases, even if the parameters identified with the IDIM of Case 1 lead to a slightly better torque estimation.

Finally, to see the improvement in terms of trajectory tracking, the tracking errors obtained when:

- Case a: a simple PD control scheme is implemented into the controller,

- Case b: a computed torque control scheme is implemented into the controller (with parameters identified in Case 1),

- Case c: a computed torque control scheme is implemented into the controller (with parameters 


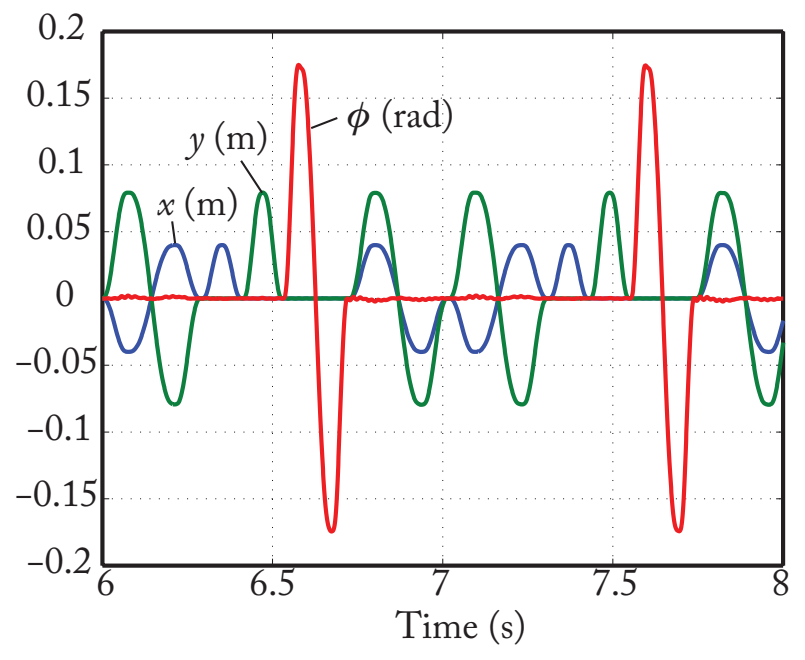

(a) Trajectory 1

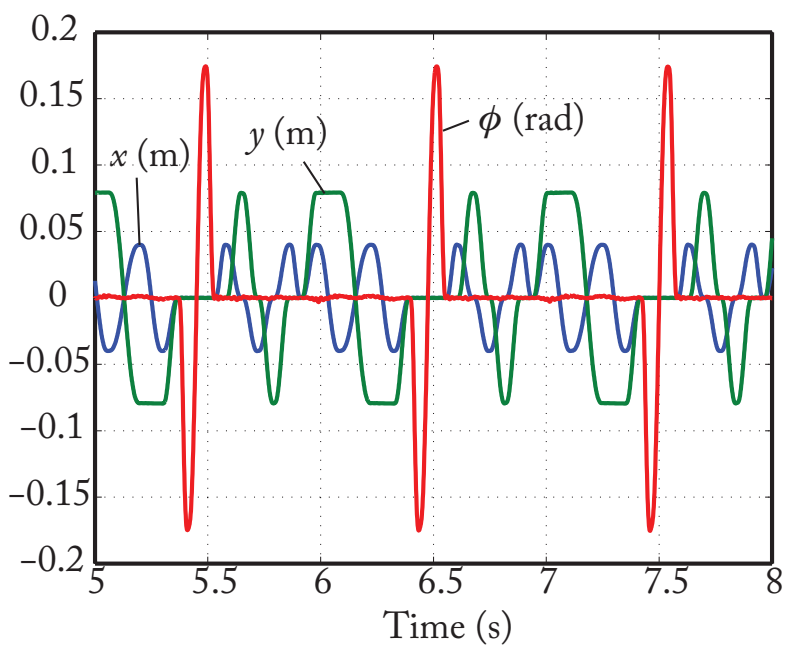

(b) Trajectory 2

Figure 7: The two trajectories used for the cross validations.

Table 3: Relative error norms (Percent).

\begin{tabular}{llllllllll}
\hline \hline & \multicolumn{1}{c}{ Trajectory 1 } & \multicolumn{7}{c}{ Trajectory 2} \\
$\hat{\chi}$ & $\tau_{1}$ & $\tau_{2}$ & $\tau_{3}$ & $\tau_{4}$ & $\tau_{1}$ & $\tau_{2}$ & $\tau_{3}$ & $\tau_{4}$ & Mean \\
\hline Case 1 & 9.1 & 8.5 & 7.5 & 8.9 & 9.8 & 8.5 & 7.6 & 10.5 & 8.8 \\
Case 2 & 9.1 & 8.5 & 8.0 & 8.6 & 10.0 & 8.6 & 8.2 & 11.7 & 9.2 \\
\hline \hline
\end{tabular}

identified in Case 2).

The maximal tracking errors for each joint are given in Table 4. Of course, when computed torque control is used, the tracking error is lower (from $54 \%$ to $76 \%$ of reduction). The comparison between the cases $\mathrm{b}$ and $\mathrm{c}$, when computed torque control is used with the two different identified dynamic parameters, is more difficult to carry out. Indeed, both controllers are quite equivalent in terms of tracking errors. Therefore, it is not possible to claim which kind of identification methodology is better.

\subsection{Discussion}

It has been observed for the DualV that, even if the results are slightly the same in terms of tracking errors, the IDIM model of Section 3.1.1 leads to better parameter estimation and torque reconstruction. However, these results are not sufficient for stating that, in general, the best IDIM to use is the one of Section 3.1.1. This should be confirmed after carrying out many experiments on different types of actuation redundant robots, and even if we had the possibility to do so, this is not the topic of the present paper. Moreover, even if for the moment we have no example in mind, we think that it could exist some particular robots for which the IDIM of Section 3.1.2 is better suited.

To our opinion, the only thing it is possible to claim is that the computation of the IDIM of Section 3.1.1 is more straightforward than the computation of the IDIM of Section 3.1.2 as all the equations are projected on the platform and as it cannot exist several combinations for projecting the equations.

\section{Conclusion}

This paper has presented a method for the identification of the inertial parameters of parallel robots with actuation redundancy. Contrary to serial robots or parallel robots without actuation redundancy 

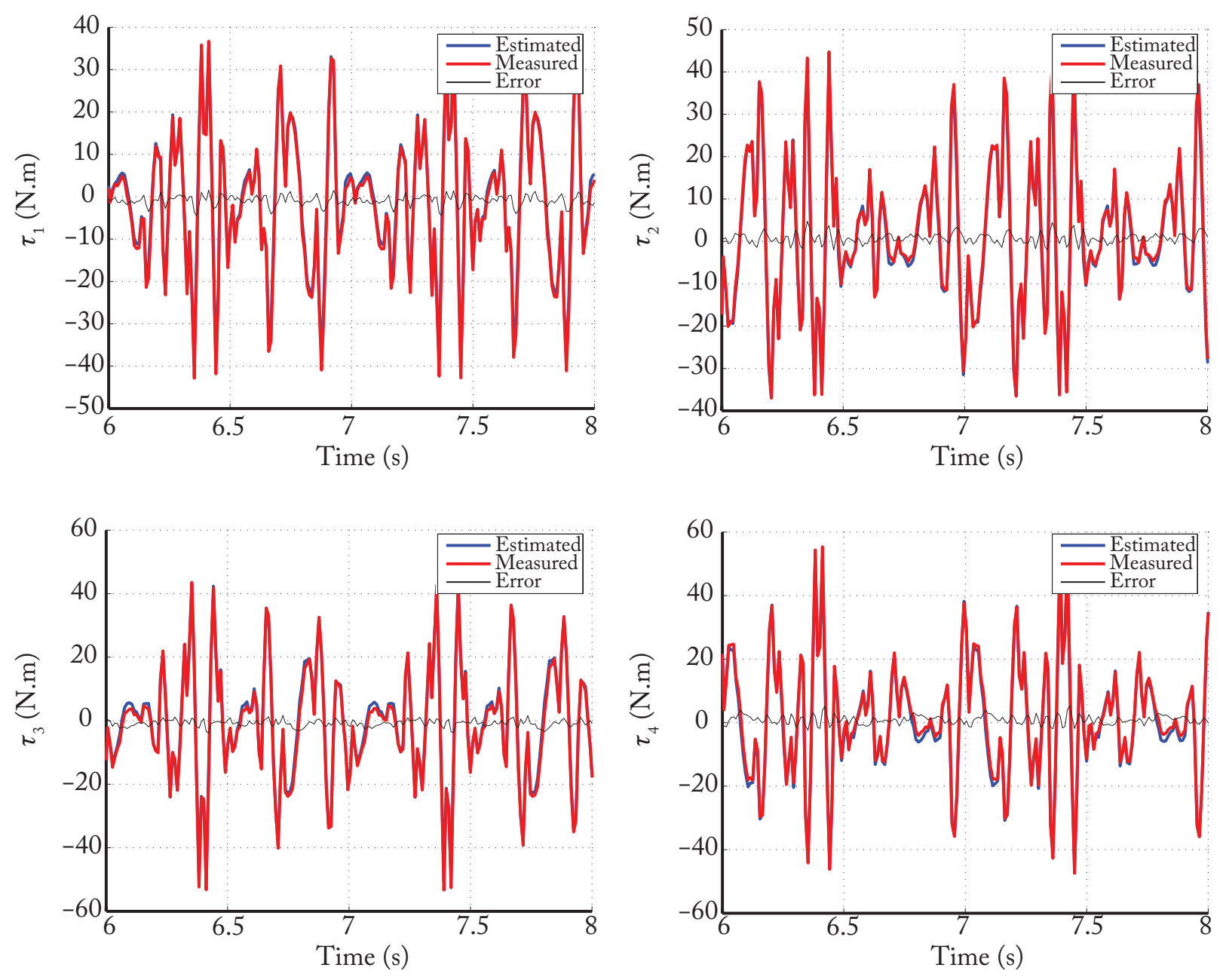

Figure 8: Measured (red lines) and estimated (blue lines) input torques rebuilt using the identified parameters of Case 1 on trajectory 1.

Table 4: Tracking errors $\left(10^{-3}\right.$ radians $)$.

\begin{tabular}{lllllllll}
\hline \hline & \multicolumn{5}{c}{ Trajectory 1} & \multicolumn{5}{c}{ Trajectory 2} \\
& $e_{1}$ & $e_{2}$ & $e_{3}$ & $e_{4}$ & $e_{1}$ & $e_{2}$ & $e_{3}$ & $e_{4}$ \\
\hline Case a & 6.35 & 6.50 & 8.00 & 8.25 & 7.70 & 7.65 & 9.40 & 7.60 \\
Case b & 2.79 & 2.15 & 1.95 & 2.10 & 2.90 & 2.65 & 2.20 & 2.05 \\
Case c & 2.80 & 2.11 & 2.06 & 2.04 & 2.83 & 2.67 & 2.25 & 2.10 \\
$\mathrm{~b} / \mathrm{a}(\%)$ & 55.9 & 66.9 & 75.6 & 74.6 & 62.3 & 65.4 & 76.6 & 73.0 \\
$\mathrm{c} / \mathrm{a}(\%)$ & 56.0 & 67.6 & 74.3 & 75.2 & 63.2 & 65.1 & 76.1 & 72.3 \\
\hline \hline & $e_{i}=\max$ & $q_{i}^{d}-q_{i}^{m} \mid$, where $q_{i}^{d}$ and $q_{i}^{m}$ are the desired and measured \\
joint $i$ positions, respectively. \\
b/a (c/a) (\%): percentage of error reduction between Cases a and b (c).
\end{tabular}

for which the dynamic identification methods are based on the use of the IDIM which calculates each joint force/torque that are linear in relation to the dynamic parameters, for actuation redundant parallel robot that are overconstrained, the usual IDIM has an infinity of solutions for the force/torque prediction, depending of the value of the desired overconstraint that is a priori unknown. As a result, the usual IDIM cannot be used as it is.

This paper proposed a universal procedure for the computation of a modified IDIM of actuation redundant parallel robots, i.e. which can be applied for any actuation redundant parallel robot 

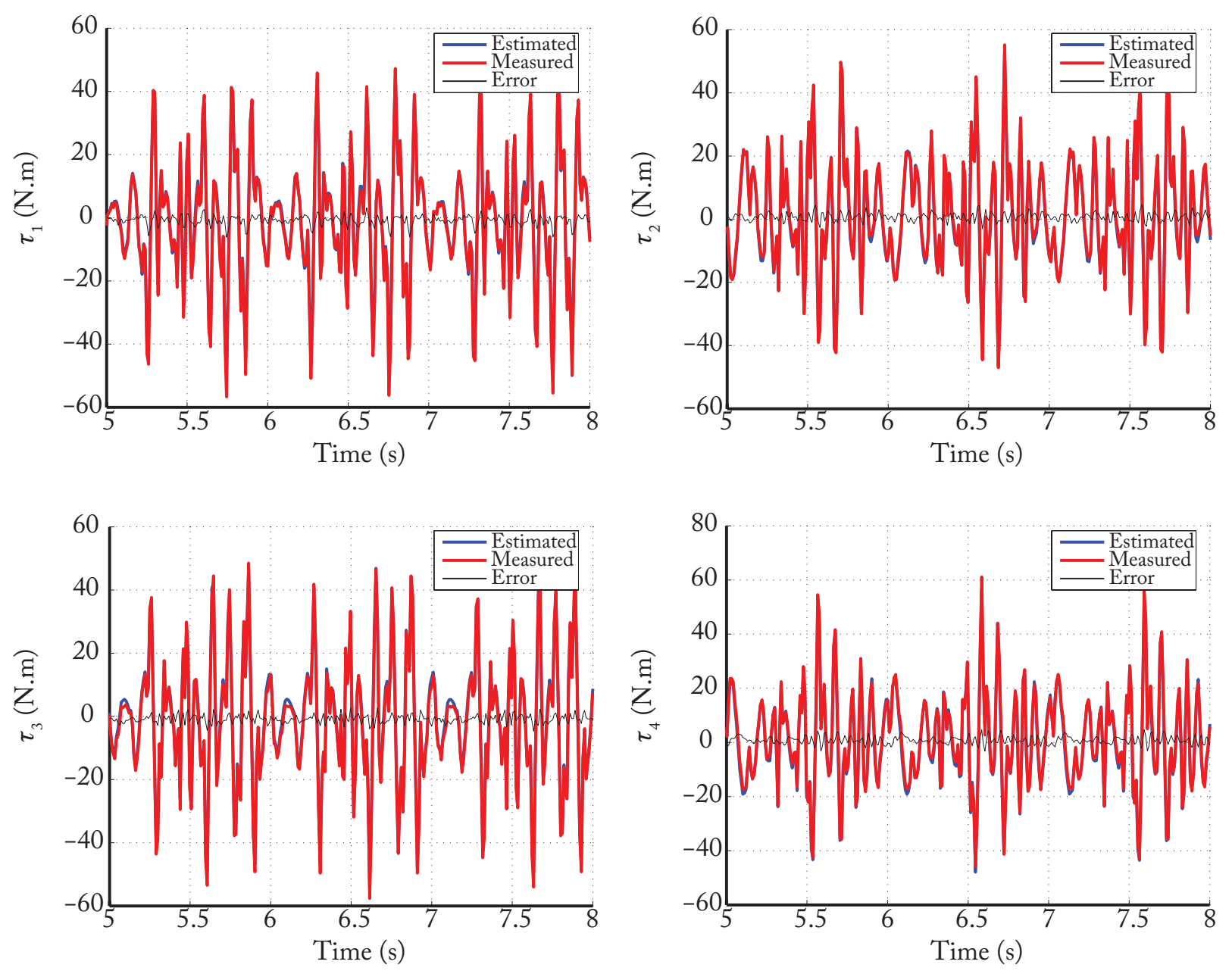

Figure 9: Measured (red lines) and estimated (blue lines) input torques rebuilt using the identified parameters of Case 1 on trajectory 2.

for which the value of the overconstraint is known or not. Two modified formulations have been shown for the IDIM of actuation redundant robots that can be used for identification purpose. This formulation consists of projecting the input torques/forces on other bodies, thus leading to unique solution of the model that can thus be used in the identification process. The identification of the inertial parameters of a planar parallel robot with actuation redundancy, the DualV, was then performed using these modified IDIM. Experimental results show that the inertial parameters of the robot were correctly identified. Moreover, for validation purpose, a known payload mass has been added on the robot to be sure that the identification process was correct. This mass has been very accurately identified. Finally, it has been shown that the torque prediction with the newly identified parameters was correct and that when the identified models were used into controller with torque prediction, the tracking errors were considerably lower.

\section{Acknowledgments}

This work has been partially funded by the French ANR project ARROW (ANR 2011 BS3 006 01).

\section{References}

[1] J.P. Merlet. Parallel Robots. Springer, 2nd edition, 2006. 
[2] B.Y. Yi, R.A. Freeman, and D. Tesar. Force and stiffness transmision in redundantly actuated mechanisms: The case for a spherical shoulder mechanism. Robotics, Spatial Mechanisms, Mechanical Systems, 45:163-172, 1994.

[3] R. Kurtz and V. Hayward. Multiple-goal kinematic optimization of a parallel spherical mechanism with actuator redundancy. IEEE Transactions on Robotics and Automation, 8(5):644-651, 1992.

[4] M. A. Nahon and J. Angeles. Force optimization in redundantly-actuated closed kinematic chains. In Proceedings of the International Conference on Robotics and Automation, 1989.

[5] A. Muller. Internal preload control of redundantly actuated parallel manipulators - its application to backlash avoiding control. IEEE Transactions on Robotics, 21(4):668-677, 2005.

[6] H. Cheng, Y.-K. Yiu, and Z. Li. Dynamics and control of redundantly actuated parallel manipulators. IEEE/ASME Trans. on Mechatronics, 8(4):483-491, 2003.

[7] M. A. Hanon. Comparison of methods for the control of redundantly-actuated robotic systems. Journal of Intelligent and Robotic Systems, 14:3-21, 1995.

[8] A. Muller. Internal prestress control of redundantly actuated parallel manipulators its application to backlash avoiding control. IEEE Transactions on Robotics, 21(4):668-677, 2005.

[9] W. Khalil, M. Gautier, and P. Lemoine. Identification of the payload inertial parameters of industrial manipulators. In Proceedings IEEE ICRA, pages 4943-4948, Roma, Italy, April 2007.

[10] C. Canudas de Wit and A. Aubin. Parameters identification of robots manipulators via sequential hybrid estimation algorithms. In Proceedings IFAC Congress, pages 178 - 183, 1990.

[11] G. Antonelli, F. Caccavale, and P. Chiacchio. A systematic procedure for the identification of dynamic parameters of robot manipulators. Robotica, 17(4):427-435, 1999.

[12] K. Kozlowski. Modelling and identification in robotics. Springer, London, 1998.

[13] J. Hollerbach, W. Khalil, and M. Gautier. Handbook of Robotics - Model Identification. Springer, 2008.

[14] W. Khalil and E. Dombre. Modeling, Identification and Control of Robots. Hermes Penton London, 2002.

[15] P.K. Khosla and T. Kanade. Parameter identification of robot dynamics. In Proceedings 24th IEEE CDC, pages 1754-1760, Fort-Lauderdale, USA, 1988.

[16] Z. Lu, K.B. Shimoga, and A. Goldenberg. Direct calculation of minimum set of inertial parameters of serial robots. Journal of Robotics Systems, 10(8):1009-1029, 1993.

[17] P.P. Restrepo and M. Gautier. Calibration of drive chain of robot joints. In Proceedings of the 4th IEEE Conference on Control Applications, pages 526-531, 1995.

[18] P. Corke. In situ measurement of robot motor electrical constants. Robotica, 23(14):433-436, 1996.

[19] M. Gautier and S. Briot. Global identification of joint drive gains and dynamic parameters of robots. IEEE Transactions on Control Systems Technology, in press, 2013.

[20] T. Hufnagel and A. Müller. A projection method for the elimination of contradicting decentralized control forces in redundantly actuated pkm. IEEE Transactions on Robotics, 28(3):723-728, 2012 . 
[21] V. van der Wijk, S. Krut, F. Pierrot, and J.L. Herder. Generic method for deriving the general shaking force balance conditions of parallel manipulators with application to a redundant planar 4-rrr parallel manipulator. In Proceedings of the 13th World Congress in Mechanism and Machine Science, Guanajuato, Mexico, June 19-25 2011.

[22] S. Briot, M. Gautier, and S. Krut. Dynamic parameter identification of actuation redundant parallel robots: Application to the dualv. In Proceedings of the IEEE/RSJ International Conference on Intelligent Robots and Systems, Tokyo Big Sight, Japan, November 3-7 2013.

[23] S. Briot and M. Gautier. Global identification of drive gains and dynamic parameters of parallel robots - part 1: Theory. In Proceedings of the 19th CISM-IFToMM Symposium on Robot Design, Dynamics, and Control (RoManSy), 2012.

[24] M. Pfurner and M.L. Husty. New Trends in Mechanism Science, chapter Implementation of a new and efficient algorithm for the inverse kinematics of serial 6R chains, pages 91-98. Springer, 2010 .

[25] S. Briot and V. Arakelian. Optimal force generation of parallel manipulators for passing through the singular positions. International Journal of Robotics Research, 27(8):967-983, 2008.

[26] D. N. Nenchev, S. Bhattacharya, and M. Uchiyama. Dynamic analysis of parallel manipulators under the singularity-consistent parameterization. Robotica, 15(4):375-384, 1997.

[27] M. Gautier. Dynamic identification of robots with power model. In Proceedings IEEE ICRA, pages 1922-1927, Albuquerque, USA, April 1997.

[28] M. Gautier. Numerical calculation of the base inertial parameters. Journal of Robotics Systems, 8(4):485-506, 1991.

[29] M. Diaz-Rodriguez, V. Mata, A. Valera, and A. Page. A methodology for dynamic parameters identification of 3-dof parallel robots in terms of relevant parameters. Mechanism and Machine Theory, 45:13371356, 2010.

[30] W. Khalil and O. Ibrahim. General solution for the dynamic modeling of parallel robots. Journal of Intelligent and Robotic Systems, 49(1):19-37, 2007.

[31] M. Gautier, P. Vandanjon, and C. Presse. Identification of inertial and drive gain parameters of robots. In Proceedings IEEE CDC, pages 3764-3769, Lake Buena Vista, FL, USA, 1994. 